Draft Version May 2, 2018

Preprint typeset using $\mathrm{L}^{A} \mathrm{~T}_{\mathrm{E}} \mathrm{X}$ style emulateapj v. 11/10/09

\title{
THE KINEMATICS OF THE GLOBULAR CLUSTER SYSTEM OF NGC 5128 WITH A NEW, LARGE SAMPLE OF RADIAL VELOCITY MEASUREMENTS
}

\author{
Kristin A. WOODLEY ${ }^{1}$ \\ Department of Physics \& Astronomy, McMaster University, Hamilton ON L8S 4M1, Canada \\ Matías Gómez \\ Departamento de Ciencias Fisicas, Facultad de Ingenieria, Universidad Andres Bello, Chile \\ WILLIAM E. HARRIS \\ Department of Physics \& Astronomy, McMaster University, Hamilton ON L8S 4M1, Canada \\ Doug Geisler \\ Departamento de Astronomía, Universidad de Concepción, Chile \\ Gretchen L. H. HARRIS \\ Department of Physics and Astronomy, University of Waterloo, Waterloo, ON, Canada \\ Draft version May 2, 2018
}

\begin{abstract}
New radial velocity measurements for previously known and newly confirmed globular clusters in the nearby massive galaxy NGC 5128 are presented. We have obtained spectroscopy from LDSS2/Magellan, VIMOS/VLT, and Hydra/CTIO from which we have measured the radial velocities of 218 known, and identified 155 new, globular clusters. The current sample of confirmed globular clusters in NGC 5128 is now 605 with 564 of these having radial velocity measurements, the second largest kinematic database for any galaxy. We have performed a new kinematic analysis of the globular cluster system that extends out to $45^{\prime}$ in galactocentric radius. We have examined the systemic velocity, projected rotation amplitude and axis, and the projected velocity dispersion of the globular clusters as functions of galactocentric distance and metallicity. Our results indicate that the metalpoor globular clusters have a very mild rotation signature of $26 \pm 15 \mathrm{~km} \mathrm{~s}^{-1}$. The metal-rich globular clusters are rotating with a higher, though still small signature of $43 \pm 15 \mathrm{~km} \mathrm{~s}^{-1}$ around the isophotal major axis of NGC 5128 within $15^{\prime}$. Their velocity dispersions are consistent within the uncertainties and the profiles appear flat or declining within $20^{\prime}$. We note the small sample of metal-rich globular clusters with ages less than 5 Gyr in the literature appear to have different kinematic properties than the old, metal-rich globular cluster subpopulation. The mass and mass-to-light ratios have also been estimated using the globular clusters as tracer particles for NGC 5128. Out to a distance of $20^{\prime}$, we have obtained a mass of $(5.9 \pm 2.0) \times 10^{11} \mathrm{M}_{\odot}$ and a mass-to-light ratio in the B-band of $16 \mathrm{M}_{\odot} / \mathrm{L}_{B \odot}$. Combined with previous work on the ages and metallicities of its globular clusters, as well as properties of its stellar halo, our findings suggest NGC 5128 formed via hierarchical merging over other methods of formation, such as major merging at late times.

Subject headings: galaxies: elliptical and lenticular, cD — galaxies: individual (NGC 5128) — galaxies: kinematics and dynamics — galaxies: star clusters — globular clusters: general
\end{abstract}

\section{INTRODUCTION}

The modern idea of galaxy formation involves hierarchically merging smaller galaxies or protogalaxies (Toomre 1977; White \& Rees 1978; Peebles 1980; White \& Frenk 1991; Kauffmann et al. 1993; Baugh et al. 1998; Cole et al. 2000; Somerville et al. 2001) that contain both baryonic and dark matter. The merging of these smaller clumps into larger systems re-

matiasgomez@unab.cl

harris@physics.mcmaster.ca

dgeisler@astro-udec.cl

glharrıs@astro.uwaterloo.ca

${ }^{1}$ Current Address: Department of Physics \& Astronomy, University of British Columbia, Vancouver BC V6T 1Z1, Canada; kwoodley@phas.ubc.ca sults in a bound system embedded in a large dark matter halo.

Globular clusters (GCs) are great tracers of the formation and evolution of their host galaxy as there is significant evidence that they trace episodes of star formation (Holtzman et al. 1992; Schweizer \& Seitzer 1993; Whitmore et al. 1993; Whitmore \& Schweizer 1995; Zepf et al. 1995; Schweizer et al. 1996; Miller et al. 1997; Carlson et al. 1998; Schweizer \& Seitzer 1998; Whitmore et al. 1999; Zepf et al. 1999; Chien et al. 2007; Goudfrooij et al. 2007; Trancho et al. 2007, among others), and generally have ages $>10$ Gyr, making these some of the oldest structures in the Universe (Kissler-Patig et al. 1998; Forbes et al. 2001; 
Puzia et al. 2002; Hempel et al. 2003; Kundu et al. 2005: Puzia et al. 2005; Strader et al. 2005; Sharina et al. 2006; Beaslev et al. 2008; Puzia \& Sharina 2008; Woodley et al. 2010, as examples). GCs are also an excellent way to study massive galaxies because they are luminous, compact and can be found in large quantities out to large galactocentric radii in relatively spherical distributions. They are also expected to retain a memory of their formation conditions (e.g. Puzia et al. 2006), so by understanding their formation we may be able to constrain formation scenarios of their host galaxy. Finally, each cluster has a unique age and abundance which are relatively straightforward to determine, providing us with the key data we need to trace their formation and chemical evolution.

The color bimodality, consisting of GCs having two distinct peaks in their broadband color histograms, corresponding to red and blue GCs, is a common feature in all early-type galaxies such as NGC 4472 (Cohen et al. 2003; Strader et al. 2007), M87 (Kundu \& Zepf 2007), and NGC 5128 (Peng et al. 2004b; Beasley et al. 2008; Woodley et al. 2010), as well as late-type galaxies, such as the Milky Way and M31 (Zinn 1985; Elson \& Walterbos 1988; Armandroff 1989; Brodie \& Huchra 1990; Zinn 1990; Harris 1991; Ashman \& Zepf 1998). The separate populations of GCs have led to the idea that they form via different mechanisms/conditions and perhaps at different times. Ashman \& Zepf (1992) proposed that red, or metal-rich, GCs can result from the merging events of gaseous disk galaxies. This type of formation suggests an age spread in the metal-rich GCs as disk mergers can continue to low redshifts. Beasley et al. (2002) and Strader et al. (2005) have proposed an interesting formation scenario that combines two scenarios proposed early: multiphase collapse (Forbes et al. 1997) which suggested that most (if not all) GCs form in their parent galaxy, but that the galaxy undergoes more than one phase of collapse and hence star formation; and the accretion scenario (Côté et al. 1998, 2000, 2002) suggesting that blue, or metal-poor, GCs are accreted into the massive galaxy via merging of surrounding satellite galaxies. Within this scenario, the metal-poor GCs could form in small protogalactic clouds in the early universe. As first suggested by Santos (2003), their formation could have been truncated by the reionization process. It is also possible that the star formation was truncated by feedback processes such as the removal of gas from supernovae driven winds (e.g. Dekel \& Woo 2003; Kauffmann et al. 2003). These protogalaxies would merge and form a massive early-type galaxy with the field stars and metal-rich GCs forming in the second collapse of star formation.

In any of the above, it is clear that age is a distinguishing factor. However, the metallicity bimodality also suggests the blue and red components may have different kinematical signatures. Kinematics can be used to test whether red and blue GCs share signatures of rotation and velocity dispersion, and how those signatures can vary with galactocentric distance. We already see in many elliptical galaxies that the red GCs are more centrally concentrated than the blue (e.g. Geisler et al. 1996; Forbes et al. 1998; Rhode \& Zepf 2001; Dirsch et al. 2003; Forbes et al. 2004; Peng et al. 2004b; Tamura et al. 2006; Woodley et al. 2007), so we question whether their kinematics also change with radius.

NGC 5128 (Centaurus A) is a moderately luminous $\left(\mathrm{M}_{B}=-21.1 \mathrm{mag}\right.$, Dufour et al. 1979) elliptical galaxy in the nearby Centaurus group, at a distance of $3.8 \pm 0.1$ Mpc (Harris et al. 2009). It has evidence for a relatively recent merger event such as a warped disk and faint shells (Malin 1978), recent star formation (Graham 1998; Rejkuba et al. 2001, 2002), and a small blue elliptical arc that appears to be a tidally disrupted stellar stream, which could be a recently accreted satellite galaxy (Peng et al. 2002). The close proximity of this galaxy allows a detailed study of its stellar population, including the GCs, that is not yet possible for other giant elliptical galaxies. However, this close proximity also means the galaxy is spread across a very wide area in the projected sky. NGC 5128 also has a low galactic latitude of $b=19^{\circ}$ so its GCs need to be securely confirmed via their radial velocities, which are generally distinct from the two forms of contamination, Milky Way foreground stars and background galaxies. Currently, over 400 GCs have been confirmed with this methodology in the past van den Bergh et al. 1981; Hesser et al. 1984, 1986; Harris et al. 1992; Peng et al. 2004b; Woodley et al. 2005; Reikuba et al. 2007; Beasley et al. 2008; Woodley et al. 2010), with a large number of these compiled in the catalog of Woodley et al. (2007).

In this paper, we confirm a number of new GCs as well as remeasure previously known GCs in NGC 5128. The observations and data reduction are presented in Section 2 followed by our presentation of radial velocity measurements in Section 3. In Section 4. we present a new kinematic analysis of the GCs in NGC 5128 as well as estimate a new mass and mass-to-light ratio of NGC 5128. We then discuss our findings in Section 5] and conclude in Section 6 .

\section{OBSERVATIONS AND DATA REDUCTION}

Our spectroscopy involves three independent observing runs, each with a primary objective to obtain radial velocity measurements of GC candidates to confirm new membership. Table 1 lists the center of field location in R. A. and Decl. (in J2000 coordinates) and the total exposure times for each field.

\subsection{LDSS-2/Baade Dataset}

We obtained multi-object slit spectroscopy with the now decommissioned Low Dispersion Survey Spectrograph-2 (LDSS-2) on the Baade/Magellan $6.5 \mathrm{~m}$ telescope at the Las Campanas Observatory in Chile. We observed 12 fields scattered around the central regions of NGC 5128 on May 9-10, 2002 (PI: D. Geisler) with the $6^{\prime}$ field of view of LDSS-2 (at the distance of NGC 5128, $1^{\prime}$ is approximately $1.1 \mathrm{kpc}$ ). Our slit sizes easily encompassed both our target objects as well as the sky immediately surrounding each object. A gain of $1.0 \mathrm{e}^{-} / \mathrm{ADU}$ and a readout noise of $5.0 \mathrm{e}^{-}$were used in this study.

Our general reduction was performed with the Image Reduction and Analysis Facility (IRAF)2 software. Each

2 IRAF is distributed by the National Optical Astronomy Observatories, which are operated by the Association of Universities for Research in Astronomy, Inc., under cooperative agreement with the National Science Foundation. 
of our target fields was taken with exposure times between 1000-1800 seconds. We obtained and combined zero (bias) frames (zerocombine) for each night and removed the bias from our science exposures. The overscan region of the CCD was also removed from our science target exposures (ccdproc), followed by the removal of cosmic rays with the European Southern ObservatoryMunich Image Data Analysis System (ESO-MIDAS) task filter/cosmic. The flat fields were combined for each field (flatcombine in IRAF) and applied to our science fields (ccdproc). Within each slit we fit, traced, and extracted each object and sky spectrum (apall). Comparison arcs, taken adjacent to field exposures, were used to generate a transformation from pixel to wavelength for each of our target objects (identify) and applied with dispcor. We used the neutral oxygen $5577 \AA$ sky line to correct for small shifts (typically less than $0.5 \AA$ ) in our spectroscopy (specshift) and then subtracted the sky from the targets (sarith) and then cleaned the spectra (lineclean) using a low rejection of 8 and a high rejection of 2.5 below and above the residual sigma with a spline3 function. Each field was observed two times and then sum combined (scombine). Our radial velocities were then measured with fxcor using GC0106 as a template spectrum. This object is a bright cluster with previously well determined radial velocity from the literature.

\subsection{VIMOS MOS/VLT Dataset}

We obtained spectroscopy with the VIMOS MOS/VLT $8 \mathrm{~m}$ telescope in 2007 (PI: M. Gómez, Program ID 079.D0539), on Paranal, Chile. We obtained 5 fields located around the inner regions of NGC 5128, each with 4 CCD quadrants of $7^{\prime} \times 8^{\prime}$ separated by $2^{\prime}$ gaps. We targetted 166 previously known GCs and the remaining slits were placed on candidate GCs. Our slit width was $1.0^{\prime \prime}$, emcompassing both our target objects as well as surrounding sky.

Our target data fields were taken in $\sim 1000$ second exposures with the high-resolution (HR) blue grism with no filter and approximate wavelength coverage of $4100-6300 \AA$. The spectral resolution was 2050 and the dispersion $0.51 \AA /$ px for a $1^{\prime \prime}$ slit. We observed with a binning of 1 by 1 and a gain of $1.96 \mathrm{e}^{-} / \mathrm{ADU}$ and a readout noise of $3.1 \mathrm{e}^{-}$.

Our general reduction performed with IRAF involved combining (zerocombine) and removing our zero (bias) exposures from the science targets along with subtracting the overscan region (ccdproc). We then subtracted the cosmic rays in our target fields with the ESO-MIDAS task filter/cosmic. Following the cosmic ray removal, we returned to IRAF to identify and fit the sky regions as well as trace and fit each target spectrum with a legendre function of order 5 . We then extracted and removed the sky spectrum from our target spectrum (apall). A comparison arc, taken with each target field, was used to identify lines (identify) from which we applied the dispersion correction to the data (dispcor). The different exposures for each field were combined (scombine) and the radial velocities were measured (fxcor) using the template GC 158-213 (Puzia et al. 2002).

\subsection{Hydra/CTIO Dataset}

We obtained spectroscopy from the Hydra Spectrograph on the Cerro Tololo Interamerican Observatory (CTIO) Blanco $4 \mathrm{~m}$ telescope, in Chile (PI: W. Harris, Program ID 2007A-0070). We observed from April 7 to April 10, 2007 and had 2.5 usable nights of data out of 4, with lost time due almost entirely to poor weather conditions. We observed 4 target fields, each with a 40 ' field. The fiber spectrograph has 2 " diameter fibers, easily capturing the light of a GC at the distance of NGC 5128. We placed available fibers on both candidate and known GCs as well as approximately 25 fibers placed on random sky positions in each field. Our sky fibers were scattered throughout the full radial range and within the full concentric range of the field.

All of our data was obtained in the red regime with a range of $\sim 2300 \AA$ centered on $7600 \AA$, capturing $\mathrm{H} \alpha$ and the two bluest of the Calcium triplet lines. We used the KPGLF grating with a dispersion of $0.57 \AA / \mathrm{px}$ and no filter. Our binning was $1 \times 2(2048 \times 4096$ pixels $)$ and we used a gain setting of $0.84 \mathrm{e}^{-} / \mathrm{ADU}$ and a readout noise of $3 \mathrm{e}^{-}$.

We reduced our data with the IRAF software. We removed the combined zerocombine bias in IRAF as well as subtracting the overscan region of the CCD from the exposures (ccdproc). The cosmic rays were removed using the L.A. Cosmic task (van Dokkum 2001). We created our flat field using Milky flats taken on the first day of observations by combining our 5 exposures (each of 300 seconds) using flatcombine. The task dohydra was then run on each target exposure to define the apertures, fit a flat field to our combined milky flat, as well as define a pixel-to-wavelength transformation with our comparison arc spectrum, which was then applied to our target data. Next, we combined our twilight exposures for each night (flatcombine) and corrected for the fibre throughput differences. During our pixel-to-wavelength correction, we also trimmed the exposures to include $6500-8740 \AA$ with 2000 pixels. We then extracted the spectra from multito one-dimensional fits file format with scopy.

In order to remove the sky contribution, we scaled the averaged sky spectrum for all fibers in each field to match the intensity of the spectrum of each individual object, which was then subtracted from the target spectrum using sarith. We removed the continuum from each exposure (continuum) with a 10th order legendre function, rejecting the 6 lowest and 3 highest pixels. Each object was then combined with the same target from all exposures with scombine by taking the average.

We measured radial velocities of our target objects by creating a template GC using two bright GCs with previously measured radial velocity measurements from highresolution spectroscopy. We chose GC0330 and GC0365 from field 1 with weighted velocity measurements of $673 \pm 1 \mathrm{~km} \mathrm{~s}^{-1}$ and $594 \pm 1 \mathrm{~km} \mathrm{~s}^{-1}$, respectively. We combined the two fully reduced, sky subtracted spectra by averaging with scombine after each spectrum had been doppler corrected to $0 \mathrm{~km} \mathrm{~s}^{-1}$. With our template in hand, we used fxcor to cross-correlate each object with our template M31 cluster GC 158-213.

3. GLOBULAR CLUSTER SYSTEM OF NGC 5128 3.1. Newly Confirmed Globular Clusters 
We have remeasured radial velocities for 218 previously known GCs, listed in Table 2. This table lists the known GC ID, the R.A. and Decl. (J2000), and the velocity measurements presented here. It also lists the new weighted velocity measurement of all GCs in the past literature (van den Bergh et al. 1981; Hesser et al. 1984, 1986; Harris et al. 1992; Peng et al. 2004c; Woodley et al. 2005; Rejkuba et al. 2007; Beasley et al. 2008; Woodley et al. 2010) including the new measurements of this study. We have remeasured 64 GCs with LDSS-2, 154 GCs with VIMOS, and 57 GCs with Hydra. Least square fits are shown for the comparison of our measured velocities with known values in the literature in Figure 1. The comparisons for nearly all GCs are well within the measurement uncertainties. In the rare cases of outlying measurements, the most obvious being GC0445 in the Hydra study, there is only one previous radial velocity measurement from Woodley et al. (2010), but both measurements are still within the bounds of accepted radial velocity confirmation of $200-1000 \mathrm{~km} \mathrm{~s}^{-1}$. We have higher confidence in the radial velocity measured with GMOS because the spectrum had a higher $\mathrm{S} / \mathrm{N}$ than the Hydra data.

Table 3 lists our 155 newly confirmed GCs from our radial velocity measurements. Table 3 lists the GC ID, the R.A. and Decl. (J2000), the $\mathrm{C}, \mathrm{M}$, and $\mathrm{T}_{1}$ magnitudes from Harris et al. (2004a), and the measured radial velocity. We identified 29 new GCs with LDSS-2 (GC0416GC0444), 80 new GC\$3 with VIMOS (GC0480-GC0559), and 46 new GCs with Hydra (GC0560-GC0605). The final column in the table, lists the weighted average of all radial velocity measurements of these new GCs.

We compare all multiple measurements of the same GC within each radial velocity study to test for consistent measurements. A comparison fit is shown in Figure 2 for the 4,1 , and 17 multiple measurements for the LDSS2, VIMOS, and Hydra studies, respectively. An average uncertainty for the studies is also indicated in the figure. We find the multiple measurements match a 1:1 fit within the measurement uncertainties of each study. The mean rms difference between multiple measurements are $64 \mathrm{~km}$ $\mathrm{s}^{-1}$ for LDSS-2, $22 \mathrm{~km} \mathrm{~s}^{-1}$ for Hydra, and $35 \mathrm{~km} \mathrm{~s}^{-1}$ for VIMOS (recall there is only $1 \mathrm{GC}$ measured in the VIMOS dataset that was measured in multiple fields).

Of our measured GC candidates there are a small number with velocities between $150-250 \mathrm{~km} \mathrm{~s}^{-1}$ which may be either GCs or foreground Milky Way stars. This velocity domain is the major region of contamination of the GC catalog in NGC 5128. To distinguish between these two objects, we measured their structural parameters using our superb $1.2 \times 1.2 \mathrm{deg}^{2}$ Magellan/Inamori Magellan Areal Camera and Spectrograph (IMACS) images ( $R$ filter) taken in $0.5^{\prime \prime}$ seeing. The structural parameters were measured with ISHAPE (Larsen et al. 1999, 2001) which convolves the stellar point spread (PSF) function with an analytical King profile (King 1962) and compares the result with the input candidate image achieving a best match. The structural parameters, measured from the models, that we used are the core

\footnotetext{
${ }^{3}$ Four of these newly identified GCs may be stars based on their structural paramter measurements, but have velocities within the accepted range. These objects, GC0485, GC0508, GC0523, and GC0526, are included here as new GCs but with caution.
}

radius, $r_{c}$, the tidal radius, $r_{t}$, the concentration parameter, $c=r_{t} / r_{c}$, and ellipticity. The half-light radii can also be obtained from the transformation, $r_{e} / r_{c} \simeq$ $0.547 c^{0.486}$ which is good to $\pm 2 \%$ for $c>4$ (Larsen et al. 2001), which is satisfied for GCs in NGC 5128 (see Gómez \& Woodlev 2007). We compared the measured structural parameters of the GC candidates to normal GCs in NGC 5128 (Harris et al. 2006: Gómez et al. 2006; Gómez \& Woodlev 2007; Mclaughin et al. 2008), as well as to a delta distribution light profile, which describes the PSF of a star. Based on their structural parameters, we classified a small handful of candidates as GCs with low velocity measurements. With our preliminary look at the structural parameters of GCs in NGC 5128, we note that five GCs are well fit by a delta distribution light profile. Four of these are newly identified here (GC0485, GC0508, GC0523, and GC0526), while the other is GC0472. A closer look at the structural parameters of these objects is underway, however, we have included them here because they satisfy the velocity criteria for the GCs in NGC 5128. We have determined the kinematics below including and excluding these 5 GCs and found that there is no kinematic difference between the two solution sets within the uncertainties. The results presented thus include the objects.

\subsection{Radial Velocity Distributions}

We have divided our globular cluster system (GCS) into either metal-poor or metal-rich GCs based on their color information. We calculated a metallicity $[\mathrm{Fe} / \mathrm{H}]_{C-T_{1}}$ obtained from a transformation of dereddened $\left(\mathrm{C}-\mathrm{T}_{1}\right)$ derived by Harris \& Harris (2002) calibrated through the Milky Way GC data. The transformation, calibrated in the range of $\left(\mathrm{C}-\mathrm{T}_{1}\right)_{o}=0.9$ to $1.9 \mathrm{mag}$ and $[\mathrm{Fe} / \mathrm{H}]=-2.3$ to -0.1 , is

$[F e / H]_{C-T_{1}}=-6.037 \times\left(1-0.82 \times\left(C-T_{1}\right)_{o}\right)+\left(0.162 \times\left(C-T_{1}\right)_{o}^{2}\right)$

where

$$
\left(C-T_{1}\right)_{o}=C-T_{1}-1.966 \times E(B-V)
$$

We use a foreground reddening value of $\mathrm{E}(\mathrm{B}-\mathrm{V})=0.11$ for NGC 5128, corresponding to $\mathrm{E}\left(\mathrm{C}-\mathrm{T}_{1}\right)=0.22$ for the transformation. The uncertainties for a typical color are \pm 0.07 dex in the metal-rich and \pm 0.2 dex in the metalpoor regimes. If there is no $\mathrm{C}-\mathrm{T}_{1}$ color for the $\mathrm{GC}$, we examine their U-V color and followed the transformation of Reed et al. (1994)

$[F e / H]_{U-V}=-3.061+(2.015 \times((U-V)-E(U-V)))$

with a foreground reddening value of $E(U-V)=$ $E(U-B)+E(B-V)=0.2$. The GCs were classified as metal-poor $\left([\mathrm{Fe} / \mathrm{H}]_{C-T_{1}}<-1.0\right)$ or as metal-rich $\left([\mathrm{Fe} / \mathrm{H}]_{C-T_{1}} \geq-1.0\right)$ (Harris et al. 2004b; Woodley et al. 2005, 2007). When neither C, T 1 , U, or $\mathrm{V}$ information is available, we divided the GCs as metal-rich if $(B-I) \geq 2.072$ and as metal-poor if $(B-I)<2.072$ following Peng et al. (2004b). Our final GC sample of 605 has 291 metal-poor, 292 metal-rich, and 22 GCs with insufficient photometry for any of the above transformations.

The radial velocity distribution functions are shown in Figure 3 for the entire GC population, the red GCs, 
the blue GCs, and the newly confirmed GCs from this study. We fit a Gaussian function to the entire GCS using RMIX]. We find the best Gaussian fit has a mean of $517 \pm 7 \mathrm{~km} \mathrm{~s}^{-1}$ and a sigma of $160 \pm 5 \mathrm{~km} \mathrm{~s}^{-1}$. The median is $v_{p}=526 \mathrm{~km} \mathrm{~s}^{-1}$ and the standard Pearson's approximation for the mode is $v_{p}=3 \times$ median $-2 \times$ mean $=545$ $\mathrm{km} \mathrm{s}^{-1}$, which is closer to the accepted systemic velocity of the galaxy of $541 \mathrm{~km} \mathrm{~s}^{-1}$. The shift to lower mean velocity than the galaxy's systemic velocity may suggest that our catalog of GCs may still contain contamination at the low velocity end from foreground blue Milky Way halo stars. We expect to find the higher velocity counterparts to these lower velocity GCs, or hope to remove the possible low velocity contamination in future work. We do not find any bias in previous radial velocity searches which would hinder finding these higher velocity GCs.

We have analyzed the radial velocity measurements as a function of galactocentric distance, shown in Figure 4 . If a large number of low-velocity objects are Milky Way foreground star contamination, we would expect to see a large number of blue objects covering this velocity range. Looking at Fig. 4, this is not clear, but in the $15^{\prime}-20^{\prime}$ range, we can see there are more GCs with lower radial velocities than high radial velocities.

To examine this more closely, we have created velocity distributions as a function of galactocentric position, shown in Figure 5. We have binned our GCs in units of effective radius of the galaxy light $\left(\mathrm{R}_{\text {eff }} \sim 5^{\prime}\right)$, from $0^{\prime}$ $5^{\prime}, 5^{\prime}-10^{\prime}, 10^{\prime}-15^{\prime}, 15^{\prime}-20^{\prime}$, with the outer GCs in the last bin of $20^{\prime}-45^{\prime}$. We fit the distributions with unimodal Gaussians with RMIX, overplotted in the figure, with mean, sigma, and reduced $\chi^{2}$ values listed in Table 4.

While it is clear that the metal-poor GCs on average lie at lower radial velocities than the metal-rich GCs, only the $10^{\prime}-15^{\prime}$ and $20^{\prime}-45^{\prime}$ bins show the metal-poor GCs lie at significantly lower velocities. They may well be affected by contamination of foreground field stars in these outer regions, but the number of known GCs here is quite low, and the asymmetries may be purely low number statistics.

\subsection{Photometry and Projected Distribution}

With a total sample of 605 GCs in NGC 5128, we briefly examine their photometric properties and projected distribution. In Figure 6 we show the color, $\mathrm{C}-\mathrm{T}_{1}$, as a function of the projected galactocentric radial distance in the galaxy. There are 4 very red GCs (GC0078, GC0408, GC0411, and GC0552) which by their colors may be red background galaxies, but their radial velocity measurements are $568 \pm 28,838 \pm 63,548 \pm 33$, and $811 \pm 32 \mathrm{~km} \mathrm{~s}^{-1}$, respectively. The 3 very blue GCs (GC0084, GC0282 and GC0432) have radial velocities of $348 \pm 31,613 \pm 6$, and $396 \pm 84 \mathrm{~km} \mathrm{~s}^{-1}$, respectively. These blue GCs could either be Milky Way foreground stars or they could genuinely be very young GCs. Ages of these blue GCs determined spectroscopically are needed to securely distinguish the difference. We exclude these 7 objects from our kinematical analysis below in an attempt to examine the kinematics of the normal GC population. We have also analyzed the kinematics after removal of

\footnotetext{
4 The complete code, available for a variety of platforms, is publicly available from Peter MacDonald's Web site at http://www.math.mcmaster.ca/peter/mix/mix.html
}

the 5 GCs that may be stars (discussed in Section 3.1), and find there is no difference of any determined parameter within uncertainties.

The GC luminosity function is considered a standard candle distance indicator. The turn-over magnitude of the GC luminosity function of the Milky Way and M31 GCSs has a mean of $V=-7.4 \pm 0.2 \mathrm{mag}$ (Harris 1991; Ashman \& Zepf 1998). With our a priori knowledge of a distance, we can therefore estimate the turnover magnitude of the GCS in NGC 5128 to be $V=20.8 \mathrm{mag}$, corresponding to $\mathrm{T}_{1}=20.3 \mathrm{mag}$. The left hand side of Figure 7 shows the luminosity function for 569 GCs with the necessary photometry. The distribution appears Gaussian with a mean of $19.44 \pm 0.04$ mag and a sigma of $1.03 \pm 0.03 \mathrm{mag}$. Therefore we are clearly missing the faintest GCs in the system. We have also fit the luminosity functions of the metal-rich and metal-poor GC subpopulations and found a mean of $19.28 \pm 0.06$ mag and a sigma of $0.90 \pm 0.04 \mathrm{mag}$ for the metal-poor GCs and a mean of $19.36 \pm 0.06 \mathrm{mag}$ and a sigma of $0.98 \pm 0.04 \mathrm{mag}$ for the metal-rich GCs. The metalrich GCs have a slightly fainter peak luminosity than the metal-poor GCs, which has also been seen in other GCSs (Larsen et al. 2001; Tamura et al. 2006). Assuming the mass distribution between the metal-rich and metal-poor GCs is the same (which may not be true if the GC subpopulations formed at different times), the difference in the turn-over magnitude would be expected if the color of a GC reflects its metallicity, which is a good approximation for GCs older than a few Gyrs (Worthey et al. 1994). The integrated light of a metal-poor GC would appear brighter than a metal-rich GC, at a given age, because the giant stars would be brighter for a metal-poor GC (Elson \& Santiago 1996).

On the right side of Fig. 7 the color-magnitude diagram of the GCS shows a clear bimodality, also seen in Fig. 6. The photometric uncertainties for the bright objects from Harris et al. (2004a,b) range typically from $0.05-0.09 \mathrm{mag}$, so much of the observed scatter in both sequences in Fig. 7 represents real cluster-to-cluster metallicity differences.

The projected radial distribution of the 605 GCs in NGC 5128 is shown as a function of projected azimuthal angle in the sky (measured in degrees east of north) in Figure 8. The GCS of NGC 5128 has been spatially searched extensively out to $10^{\prime}$, while only a few searches, including the work presented here, have tried to search thoroughly out to $20^{\prime}$. Beyond this distance, only Peng et al. (2004c) has made an attempt to extensively search along the isophotal major axis of the galaxy of $35^{\circ} \mathrm{E}$ of N (Dufour et al. 1979). Within 20', we do see a lack of GCs along the isophotal minor axis $\left(119^{\circ} \mathrm{E}\right.$ of $\mathrm{N}$ Dufour et al. 1979). However, with upcoming searches for GCs beyond 20', we will be able to test if the GCS follows the ellipticity of the underlying galaxy light.

\section{THE KINEMATICS AND DYNAMICS OF THE GLOBULAR CLUSTER SYSTEM}

\subsection{Mass and Mass-to-Light Determinations}

In the current paradigm of cold dark matter (CDM) and hierarchical merging, we expect to find luminous, baryonic matter at the centers of much larger dark matter halos. Determining the full extent of these dark matter halos and the total mass of a galaxy has proven to be 
quite challenging.

The technique of measuring integrated stellar light spectroscopy is useful for obtaining a velocity dispersion from high $\mathrm{S} / \mathrm{N}$ data in which one can calculate a mass. However, the stellar halo gets fainter with galactocentric distance, limiting studies to a few inner effective radii (Kronawitter et al. 2000; Gerhard et al. 2001). HI gas has also been successfully used to determine mass content, but is inapplicable for early-type galaxies without large amounts of gas. Extended X-ray halos are found in massive early-type galaxies (Mathews \& Brighenti 2003; Humphrey et al. 2006) which may be used to determine the mass out to large distances, where dark matter is expected to dominate. This has been done in luminous ellipticals such as in NGC 4486 (M87) (McLaughlin 1999), and NGC 4649 (M60) (Bridges et al. 2006; Hwang et al. 2008).

In NGC 5128, tracer particles have been used to estimate the mass via both $\mathrm{PNe}$ (Peng et al. 2004a; Woodley et al. 2007) and GCs (Peng et al. 2004b; Woodley 2006; Woodley et al. 2007). With our larger sample of over 560 GCs with radial velocity measurements, we can derive an improved estimate of the mass and mass-to-light ratio of NGC 5128.

\subsubsection{Pressure and Rotation Supported Mass}

We calculate the mass of NGC 5128 using its GC population by adding together the component of mass supported by pressure, $M_{p}$, and that supported by rotation, $M_{r}$ with

$$
M_{t o t}=M_{p}+M_{r}
$$

where $M_{p}$ is determined by the Tracer Mass estimator, discussed by Evans et al. (2003) as

$$
M_{p}=\frac{C}{G N} \sum_{i}\left(v_{f_{i}}-v_{s y s}\right)^{2} R_{i}
$$

where $N$ is the number of objects in the sample, $R_{i}$ is the projected galactocentric radius of the tracer object, and $v_{f_{i}}$ is the radial velocity of the tracer object with the rotation component removed. We have determined the rotational component for the GCs using the kinematic solution described in Section 4.1.2. The Tracer Mass estimator is an advantageous mass estimator over the projected and virial mass estimators described in Bahcall \& Tremaine (1981) and Heisler et al. (1985) because it does not assume that the distribution of tracer objects follows the underlying dark matter halo.

We assume the GCs are an isotropic population, so the value of $\mathrm{C}$ is

$$
C=\frac{4(\alpha+\gamma)(4-\alpha-\gamma)\left(1-\left(\frac{r_{\text {in }}}{r_{\text {out }}}\right)^{(3-\gamma)}\right)}{\pi(3-\gamma)\left(1-\left(\frac{r_{\text {in }}}{r_{\text {out }}}\right)^{(4-\alpha-\gamma)}\right)}
$$

where $r_{\text {in }}$ and $r_{\text {out }}$ are the 3-dimensional radii corresponding to the 2-dimensional projected radii of the innermost, $R_{i n}$, and outermost, $R_{\text {out }}$, tracers in the sample. The parameter $\alpha$ is set to zero for an isothermal halo potential in which the system has a flat rotation curve at large distances. The slope of the volume density distribution is $\gamma$ which is found by determining the surface density slope of the sample and deprojecting the slope to three-dimensions, written as $r^{-\gamma}$. Although the
Tracer Mass estimator uses a sample of GCs defined between $r_{\text {in }}$ and $r_{\text {out }}$, it determines the total enclosed mass within the outermost point.

The mass component supported by rotation in Eqn. 4 is determined from the rotational component of the Jeans Equation,

$$
M_{r}=\frac{R_{\text {out }} v_{\max }^{2}}{G}
$$

where $R_{\text {out }}$ is the outermost tracer projected radius in the sample and $v_{\max }$ is the rotation amplitude.

\subsubsection{Systemic Velocity, Rotation Amplitude, and Rotation Axis}

To obtain the amount of rotation that needs to be removed from each GC in Equation 5 , we use the equation

$$
v_{p}(\Theta)=v_{\text {sys }}+\Omega R \sin \left(\Theta-\Theta_{o}\right)
$$

described in Côté et al. (2001). With our known quantities, the measured radial velocity, $v_{p}$, and the angular position of each GC measured on the projected sky in degrees East of North, $\Theta$, we fit our GC dataset with a weighted least squares fit with the non-linear function. From the fit, we extract the systemic velocity, $v_{s y s}$, the rotation amplitude (a product of the projected angular velocity and the projected galactocentric radius of the GC) $\Omega R$, and the projected rotation axis, $\Theta_{o}$, also measured in degrees East of North in the projected sky. Eqn. 8 assumes spherical symmetry, that $\Omega$ is only a function of the projected radius, and that the rotation axis lies in the plane of the sky.

\subsubsection{Surface Density Profiles}

In order to calculate Equation 6, we require the surface density profile of the GCS. GCSs typically have radial surface density profiles that fall off with a power law, with an exponent that varies between $1.0-2.5$ (Ashman \& Zepf 1998). To calculate the surface density slope of the GCS in NGC 5128, shown in Figure 9, we bin the GCs into circular annuli with equal number of GCs in each to allow near equal statistical weighting (Maíz Apellániz \& Úbeda 2005). We then fit the data with a power-law within the region of approximate azimuthal completeness between $5^{\prime}-20^{\prime}$ for the entire GCS, the metal-rich and metal-poor subpopulations of GCs. Within $5^{\prime}$, a flattening of the GCS is evident in Fig. 9 due to the lack of GC candidates in the dustlane of the galaxy. Also evident is a drop-off in the GCS beyond $20^{\prime}$ where there are few to no known GCs along the isophotal minor axis. Our best fits within these bounds yield a volume density slope of $3.38 \pm 0.17,3.26 \pm 0.29$, and $3.56 \pm 0.21$ for the GCS, the metal-poor GCs, and the metal-rich GCs, respectively, which are used in Equation 6 for all subsequent mass determinations. We also determine the volume density profile of the PNe within the $5^{\prime}-20^{\prime}$ bounds to yield a slope $3.47 \pm 0.12$ (obtained similarly in Woodley et al. 2007, who determined the surface density profile excluding only $\mathrm{PNe}$ within $5^{\prime}$ ).

\subsubsection{Mass and Mass-to-Light Results}

Following a similar technique to Schuberth et al. (2009), we have used the Tracer Mass Estimator as a way to remove GCs that have extreme radial velocities 
at a large projected distances which would artificially inflate the total mass estimate. This can be seen by their contribution to the $v^{2} R$ term in Equation 5. First, we determine the total mass of the NGC 5128 using all of the GCs in our sample. Then, we remove the GC with the highest $v^{2} R$ contribution from our sample and recalculate the total mass. We continue to calculate the total mass while removing the subsequent largest $v^{2} R$ contributor in each iteration. For each step, we determine the change in total mass from the total mass determined using the entire GCS, divided by the total number of GCs removed. Our results are shown in Figure 10. We have removed the 8 largest $v^{2} R$ contributors from the total mass estimate, as well as in the kinematic analysis that follows. These $8 \mathrm{GCs}, 4$ of which are metal-rich, are all found at a galactocentric radius greater than $16^{\prime}$, and lead to an overestimate in the mass of NGC 5128.

To generate a mass profile within NGC 5128 using its GCS, we determine the rotation and pressure supported masses in cumulative bins from $0^{\prime}-5^{\prime}, 0^{\prime}-10^{\prime}, 0^{\prime}-15^{\prime}, 0^{\prime}-20^{\prime}$, $0^{\prime}-45^{\prime}$. The results are tabulated in Table 5] with columns displaying the GC subgroup, the radial range of included GCs, the number of GCs per radial bin, the systemic velocity, the rotation amplitude, the rotation axis, the pressure supported mass, the rotation supported mass, and the last column indicates the total mass of the galaxy. The total mass determined is the mass enclosed within the outermost tracer point.

We also calculate the mass of NGC 5128 by excluding the GCs located within $0-5^{\prime}$ projected galactocentric radius listed in Table 5. The Tracer Mass estimator assumes spherical symmetry of the system and the GCs within $5^{\prime}$ have severe spatial bias within the dustlane. While we examine the enclosed mass out to $45^{\prime}$, our most secure mass estimate is out to $20^{\prime}$ (determined with the GCs between $\left.5^{\prime}-20^{\prime}\right)$ to be $(5.9 \pm 2.0) \times 10^{11} \mathrm{M}_{\odot}$. We also determine the total mass using only the metal-poor and metal-rich GCs out to $20^{\prime}$ and out to $45^{\prime}$, also listed in Table 5. Both metallicity subpopulations provide good agreement for the mass estimate to the entire GCS.

The mass-to-light ratio of NGC 5128 was also calculated using the GCS. We have calculated the absolute B-band magnitude assuming a distance of $3.8 \mathrm{Mpc}$ and an apparent B-band magnitude of 7.84 and a galactic extinction of 0.5 for NGC 5128 (Karachentsev et al. 2002). We determine the B-band mass-to-light ratio, $\mathrm{M} / \mathrm{L}_{B}$, out to $20^{\prime}$ and to $45^{\prime}$ to be $16 \mathrm{M}_{\odot} / \mathrm{L}_{B \odot}$ and $30 \mathrm{M}_{\odot} / \mathrm{L}_{B \odot}$, respectively.

\subsubsection{Comparison to Previous Measurements}

In NGC 5128, the HI gas shells were used to estimate a mass of $2 \times 10^{11} \mathrm{M}_{\odot}$ within $15 \mathrm{kpc}$ under their assumption that NGC 5128 is at a distance of 3.5 Mpc (Schiminovich et al. 1994). With the distance of $3.8 \mathrm{Mpc}$ used in this study, the mass determined by Schiminovich et al. (1994) would increase to just less than $2.2 \times 10^{11} \mathrm{M}_{\odot}$ within $15 \mathrm{kpc}\left(\right.$ or $13.8^{\prime}$ ). Within $13^{\prime}$ we re-evaluate our total mass estimate and find $M_{t}=(3.5 \pm 1.2) \times 10^{11} \mathrm{M}_{\odot}$, in agreement with the HI gas shells estimate.

Peng et al. (2004b) have used 215 GCs extending out to $40 \mathrm{kpc}\left(\sim 36^{\prime}\right)$ to estimate a pressure supported mass of $7.5 \times 10^{11} \mathrm{M}_{\odot}$ (see Woodley et al. 2007, for this mass, corrected from Peng et al. (2004b)). We reevaulate the pressure supported mass out to $36^{\prime}$ and obtain $M_{p}=(9.7 \pm 3.3) \times 10^{11} \mathrm{M}_{\odot}$, matching the estimate of Peng et al. (2004b) within uncertainties.

Woodley et al. (2007) found a total mass, including the contribution from rotation and pressure, of $(1.3 \pm 0.5) \times$ $10^{12} \mathrm{M}_{\odot}$ from $340 \mathrm{GCs}$ out to the full extent of the known $\operatorname{GCS}\left(45^{\prime}\right)$. We find $M_{t}=(1.1 \pm 0.4) \times 10^{12} \mathrm{M}_{\odot}$ in excellent agreement with this result although we use a much larger sample of $429 \mathrm{GCs}$ (excluding GCs with $R_{a c}<5^{\prime}$ ).

Both Peng et al. (2004b) and Woodley et al. (2007) have used samples with quite low number of GCs beyond $10^{\prime}$ in galactocentric radius. We do, however, find good agreement with their earlier work, but with better constraints on the uncertainties associated with the determined mass.

\subsection{Kinematic Analysis}

The previous kinematic studies of the GCS in NGC 5128 have been done with smaller samples of GCs, with 215 GCs used in Peng et al. (2004b) and 340 GCs used in Woodley (2006) and Woodley et al. (2007). Here, with a larger sample size and better spatial coverage, we evaluate the kinematics of the GCS.

We determine the kinematics for the GCs using Equation 8 for the full sample or discretely binned subsamples by weighting the individual GCs according to their individual radial velocity uncertainties, $v_{p, e r r}$ and the random velocity component of the system, $v_{\text {ran }}$. The radial velocities and associated uncertainties used in this study are the weighted averages of all previous measurements. The random velocity component is the standard deviation of the radial velocities of the GCs from the best fit rotation curves determined without any weighting. The final uncertainty for each GC used in Eqn. 8 is $\sigma=\left(v_{p, e r r}^{2}+v_{\text {ran }}^{2}\right)^{1 / 2}$, and the weight is $1 / \sigma^{2}$. The dominant uncertainty is $v_{\text {ran }}$ providing all GCs a near equal weighting in the fitting.

Our initial results are shown in Figure11 for the entire GCS, and for the metal-poor and metal-rich subpopulations of GCs. The best sine curve fits clearly show the large dispersion (or random motion) of the GCS with a small rotational component. Our results are presented in Table 6] which lists the GC subgroup, the radial range of the $\mathrm{GC}$ coverage, the average radius, and number of GCs within the subgroup, followed by the best fit systemic velocity, rotation amplitude, rotation axis, the isophotal major axis of $215^{\circ} \mathrm{E}$ of $\mathrm{N}$ (Dufour et al. 1979) subtracted from the rotation axis, velocity dispersion, and rotation parameter (rotation amplitude/velocity dispersion). The latter two will be discussed in Section 4.2.1. We note the kinematics presented in Table 5, while calculated with the same method as in Table 6. differ by their radial ranges. In the mass determination presented in Section 4.1, we were developing a mass profile that with the inclusion of more datapoints reduces the uncertainties on the mass determined using the enclosed data. Here, we are searching for the kinematic signatures at distinct radial positions.

For the entire GCs, our best fits are $v_{\text {sys }}=517 \pm 7$ $\mathrm{km} \mathrm{s}^{-1}, \Omega R=33 \pm 10 \mathrm{~km} \mathrm{~s}^{-1}$, and $\Theta_{o}=185 \pm 15^{\circ} \mathrm{E}$ of $\mathrm{N}$. Also, our best fits for the overall kinematics are $v_{\text {sys }}=506 \pm 9 \mathrm{~km} \mathrm{~s}^{-1}, \Omega R=26 \pm 15 \mathrm{~km} \mathrm{~s}^{-1}$, and $\Theta_{o}=$ 
$177 \pm 28^{\circ} \mathrm{E}$ of $\mathrm{N}$ for the metal-poor and $v_{\text {sys }}=526 \pm 10$ $\mathrm{km} \mathrm{s}^{-1}, \Omega R=43 \pm 15 \mathrm{~km} \mathrm{~s}^{-1}$, and $\Theta_{o}=196 \pm 17^{\circ} \mathrm{E}$ of $\mathrm{N}$ for the metal-rich GC subpopulations. As discussed in Section 3.2, the overall velocity of the metal-poor GC subpopulation is shifted to a lower value than the metalrich GC subpopulation, however, both are lower than the systemic velocity of the galaxy. The rotation amplitude as first indicated in Fig. 11] is quite low. The metal-poor GC subpopulation has very mild rotation and the metalrich GCs have mild to moderate rotation as a whole. The metal-rich GC subpopulation appears to rotate around the isophotal major axis of the galaxy within $15^{\prime}$, while the metal-poor GC subpopulation does not appear to follow either the isophotal minor or major axis. We note there is no difference in the results within uncertainties if $v_{s y s}$ is fixed at the systemic velocity of the galaxy or left free in Eqn. 8.

We create kinematic profiles for the entire GCS as well as the metal-poor and metal-rich GC subpopulations as functions of galactocentric radius. We bin the GCs in galactic effective radial bins of $0^{\prime}-5^{\prime}, 5^{\prime}-10^{\prime}, 10^{\prime}-15^{\prime}, 15^{\prime}$ $20^{\prime}$, and the remaining GCs in the final bin of $20^{\prime}-45^{\prime}$ for the entire GCS and the metal-poor GC subpopulation. The metal-rich GC subpopulation has a more spatially centrally concentrated system so we bin in $0^{\prime}-5^{\prime}, 5^{\prime}-10^{\prime}$, $10^{\prime}-15^{\prime}$ bins, with the remaining metal-rich GCs in the final bin of $15^{\prime}-45^{\prime}$.

We list the results in Table 6 for the best fit systemic velocity, rotation amplitude, and rotation axis for the entire GCS, as well as metal-poor and metal-rich GC subpopulations. With radial distance, we see both the metal-rich and metal-poor populations maintain a relatively constant amount of rotation with radial distribution, with the metal-rich GCs having a larger rotation amplitude than the metal-poor GCs. Note that the rotation amplitude for the entire metal-poor GC subpopulation from $0^{\prime}-45^{\prime}$ is less than most of the values determined in the specific radial bins, which at first sight is an anomaly. However, this curious result is connected with the solutionos for the rotation axis angles, which differ by large amounts from bin to bin. When the rotation amplitude is zero, the rotation axis is ofcourse undefined, so when we average together the results from the different radial bins (all with a different axis of rotation), they average down to zero with no meaningful axis. We conclude from this that the rotation in the metal-poor GC subpopulation is very mild. There is an indication that the metal-poor GCs have an increased amount of rotation in the outer regions (beyond $20^{\prime}$ ), but again, the GC population in this region clearly violates the basic assumption of spherical symmetry used in Eqn. 8. We therefore caution as to its validity. If the metal-poor GC system rotation does increase, this may support the formation mechanisms of disk-disk mergers for NGC 5128. Here, the orbital angular momenta of the progenitor galaxies are converted to intrinsic angular momentum in the remnant from the merging event (Bekki et al. 2005). This angular momentum is transferred to the outer regions of the galaxy, and thus it would be expected for the rotation amplitude of the GCS to increase at larger radii.

The metal-poor GC subpopulation shows a potential twist in its rotation axis within the inner $5^{\prime}$ from rotation around the isophotal minor axis to around the isophotal major axis. Beyond $10^{\prime}$ the axis may change again. However, given the near zero rotation amplitude of the metal-poor GCs, it does not appear that they rotate around a well-defined rotation axis. In the inner $20^{\prime}$ the metal-rich GCs are consistent with rotation around the isophotal major axis of the galaxy. In the outer regions, the rotation axis of the metal-rich GC subpopulation appears to decrease slightly, but again, this is a region of uncertainty.

\subsubsection{Velocity Dispersion}

The velocity dispersion of the system is determined using a maximum likelihood dispersion estimator discussed in Pryor \& Meylan (1993),

$$
\sum_{i=1}^{N} \frac{\left(v_{i}-v_{\text {sys }}\right)^{2}}{\left(\sigma_{v p}^{2}+v_{p, e r r_{i}}^{2}\right)^{2}}=\sum_{i=1}^{N} \frac{1}{\left(\sigma_{v p}^{2}+v_{p, e r r_{i}}^{2}\right)}
$$

where $N$ is the number of GCs in the sample, $v_{i}$ is the radial velocity of the $\mathrm{GC}$ after subtraction of the rotational component determined in Eqn. 8 with $v_{\text {sys }}$ held constant at $541 \mathrm{~km} \mathrm{~s}^{-1}$, and $v_{p, e r r_{i}}$ is the uncertainty in the velocity measurement. The projected velocity dispersion, $\sigma_{v p}$, is determined by iteration until satisfying Equation 9] The systemic velocity used here was determined from Eqn. 8. The uncertainties in the velocity dispersion are calculated from the variance of the dispersion, listed in Prvor \& Mevlan (1993). This has been shown by Pryor \& Meylan (1993) to estimate the uncertainty to $5 \%$ with as little as 20 objects in the sample.

We list the velocity dispersions in Table 6. As a whole the metal-poor and metal-rich GC subpopulations have identical velocity dispersions within uncertainties of $149 \pm 4 \mathrm{~km} \mathrm{~s}^{-1}$ and $156 \pm 4 \mathrm{~km} \mathrm{~s}^{-1}$, respectively. However, the behaviour with galactocentric radius may be different for the different metallicity subpopulations. The metal-poor GC velocity dispersion is flat or declining out to $20^{\prime}$ beyond which it appears to rise. The metalrich GC profile shows a declining velocity dispersion out to $5^{\prime}$ after which it appears to flatten, which may indicate the metal-rich GCs are more radially biased than the metal-poor GCs. A rise in the velocity dispersion in the outer regions of a galaxy could be an indicator for large amounts of dark matter or of anisotropic orbits of the velocity ellipsoid of the GCS itself. With an extended X-ray gas profile to trace the mass out to regions beyond the halo light, we could determine the degree of anisotropy of these orbits, if they exist, using a Jeans solution. This has been done for M87 (Côté et al. 2001), M49 (Côté et al. 2003), M60 (Hwang et al. 2008), and NGC 1407 (Romanowksy et al. 2009) as examples. It may be possible to find indications of anisotropy in the GCS by examining its radial velocity as a function of radius. To analyse the ellipsoid distributions, it is more instructive to remove the rotational component of the GC subpopulations; however, considering the rotation amplitudes are nearly negligible, we use Fig. 5 as a first glance into possible anisotropy of the GCS.

If the GCs are moving on very radial orbits, we expect the distribution to be strongly peaked at the systemic velocity, while if they are moving on very circular orbits, the distribution would have a more flattened shape. Isotropic orbital motion would have a Gaussian distribution. The radial velocity distributions do not appear to 
be tightly peaked in any histogram in Fig. 5, but there is a slight tendency for the GC velocity histograms to have a wider distribution at larger galactocentric radius, thus a flattening effect. In Table 4 we quote the reduced $\chi^{2}$ values for these Gaussian fits. We note a general trend for larger $\chi_{r e d}^{2}$ values for the outer bins, indicating a poorer fit of the model to the data, perhaps by the lower number of GCs in the outermost regions. A flattened distribution may also be caused by the contribution of GCs from accreted satellite galaxies in the outer regions of the galaxy (Bekki et al. 2003). Evidence for potential substructure has been presented in M31 (Merrett et al. 2003), M87 (Doherty et al. 2009), NGC 1399 (Schuberth et al. 2009), and perhaps in NGC 1407 (Romanowksy et al. 2009). Interestingly, numerical simulations have suggested that tracer populations should have isotropic orbits in the inner regions of their host galaxy, while in the outer regions, their orbits should become anisotropic (Dekel et al. 2005; Diemand et al. 2005; Abadi et al. 2006; Gnedin \& Prieto 2006). In relation to the $\mathrm{GC}$ results presented here, the presence of substructure of accreted satellites or interlopers will bias the velocity dispersion to higher values. This is seen in the outer-most bin in the metal-poor GC subpopulations, possibly indicating substructure from an accreted satellite or Milky Way halo star contamination.

The rotation parameter is a measure of the maximum rotation divided by the velocity dispersion in a system. We obtain rotational parameters of $0.22 \pm 0.07$, $0.17 \pm 0.09$, and $0.28 \pm 0.10$ for the entire GCS, metalpoor, and metal-rich subpopulations of GCs, respectively (listed in Table 6). These values reinforce what we have already discovered about the GCS. While the velocity dispersion of the entire metal-poor and entire metal-rich GC subpopulations is consistent within uncertainties, the metal-rich GCS, as a whole, has more rotation.

\subsubsection{Kinematics for Globular Clusters with Ages}

Woodley et al. (2010) have estimated ages, metallicities, and $\alpha$-to-Fe abundance ratios for 72 GCs in NGC 5128. By calibrating their high signal-to-noise spectroscopy taken with Gemini-S/GMOS to the Lick index system (Burstein et al. 1984; Worthey et al. 1994; Worthey \& Ottaviani 1997; Trager et al. 1998), they extracted their estimates by comparing their measured indices to the simple stellar population models of Thomas et al. (2003, 2004). Using Eqn. 8, we have estimated the kinematic properties of these $72 \mathrm{GCs}$, as well as subsets of this group, such as their GCs estimated to be old (ages $\geq 8$ Gyr), intermediate $(5-8$ Gyr), and young (age $<5$ Gyr). We also analyse the old GCs in subgroups of metal-rich and metal-poor. Our results are presented in Table 7 , which lists our subgroup of GCs, the number of GCs in each subgroup, the systemic velocity, the rotation amplitude, and the rotation axis. The kinematics for the GCs subdivided by metallicity and age have quite large uncertainties because they are small samples. They do, however, indicate general properties of these small subsets of GCs. As expected, the old metal-poor GCs and old metal-rich GCs generally match the kinematic properties of the entire metal-poor GCs and metal-rich GCs, respectively. The rotation axis of the young GCs, which are all metal-rich, is quite different than that of the entire metal-rich GC population.
With a rotation axis of $80 \pm 84^{\circ} \mathrm{E}$ of $\mathrm{N}$ at the average projected radius of $5.7^{\prime}$ this is significantly different from the metal-rich GC rotation axis of $244 \pm 27^{\circ} \mathrm{E}$ of $\mathrm{N}$ at an average radius of $3.49^{\prime}$ or $178 \pm 22^{\circ} \mathrm{E}$ of $\mathrm{N}$ at an average $7.23^{\prime}$ distance. The young metal-rich GCs appear to be rotating either along the isophotal major axis, but in the opposite direction to the metal-rich GC subpopulation, or along the isophotal minor axis, or anywhere in between. In any case, their rotation axis is quite different from the bulk of the metal-rich GCs.

\subsubsection{Kinematics for Globular Clusters versus Magnitude}

The kinematics of the GCS with respect to $\mathrm{T}_{1}$ magnitude has also been analyzed upon removal of GCs that have extreme colors (see Section 3.3). The GCs are binned in magnitudes between 16-18, 18-18.5, 18.5-19, $19.5-20,20.5-23$ mags, in order to keep the number of objects per bin above a minimum of 25 . We plot the results for the systemic velocity, rotation amplitude, rotation axis, and velocity dispersion in Figure 12. We find there are no major differences in the kinematic properties of the GCs with changing magnitude. It does appear, however, that the GCs between 18 - 18.5 magnitudes have a significantly different rotation axis $\left(41 \pm 34^{\circ} \mathrm{E}\right.$ of $\mathrm{N})$ than the remaining GCs which have an axis of $\sim 200^{\circ}$ $\mathrm{E}$ of $\mathrm{N}$. We have examined the projected distribution of the GCs in this bin compared to the other magnitude bins and see no distinct difference. We also notice that the brightest GCs have a significantly larger velocity dispersion than the remaining GCs, indicating there may be evidence for varying kinematics as a function of magnitude. A similar finding was identified in NGC 1407 (Romanowksy et al. 2009); however, as they indicated, it could be due to spatial bias of the brighter objects in the sample being close to the center of the galaxy. This is not as obvious in the case of NGC 5128, as almost all GCs in this study are centrally concentrated within $15^{\prime}$.

\subsubsection{Kinematics of the Planetary Nebulae}

The PNe are a field star population at one of the latest stages of stellar evolution and are easily identified by their emission of [OIII]. Follow-up spectroscopy can then be performed on the $\mathrm{PNe}$ to measure their radial velocities which has been done for 780 PNe in NGC 5128, extending out to over $80^{\prime}$, and compiled in Peng et al. (2004a).

The kinematic properties of the 780 PNe are investigated in Woodley et al. (2007) in the same manner as the GCs presented in this study. They found the PNe had a rotation amplitude of $76 \pm 6 \mathrm{~km} \mathrm{~s}^{-1}$, a rotation axis of $170 \pm 5^{\circ} \mathrm{E}$ of $\mathrm{N}$, and a velocity dispersion of $118 \pm 13 \mathrm{~km}$ $\mathrm{s}^{-1}$. The rotation amplitude of the $\mathrm{PNe}$ is a bit larger than the GCS as a whole and the rotation axis of the $\mathrm{PNe}$ is quite uniform with increasing radius (see Table 5 in Woodley et al. 2007). The rotation axis is also, interestingly, similar to the isophotal major axis of the galaxy, closely mimicing the metal-rich GC subpopulation in the inner $15^{\prime}$. The velocity dispersion of the PNe is different than the GCs. The PNe are dynamically colder in the inner $5^{\prime}$ and their velocity dispersion continues to decline with increasing galactocentric radius.

Declining velocity dispersions of the $\mathrm{PNe}$ with galactocentric radius have been found for NGC 3379 (Romanowsky et al. 2003; Douglas et al. 2007), 
NGC 821 (Romanowsky et al. 2003), NGC 4494 (Romanowskv et al. 2003; Napoliano et al. 2009), NGC 5128 (Peng et al.|2004a; Woodlev et al.|2007), and NGC 4697 (Méndez et al. 2009), which could indicate a lack of dark matter in the system (Romanowsky et al. 2003). However, GCSs studied within NGC 3379 (Pierce et al. 2006) have shown an increasing velocity dispersion, indicating there is likely to be some amount of dark matter present. Recent work has shown that PNe may be on radial orbits (Dekel et al. 2005; Mamon \& Lokas 2005; Abadi et al. 2006), which may also cause the declining velocity dispersions. Anisotropy may not necessarily be expected of the GCs because these two populations trace different kinematical histories of their host galaxies, especially if the galaxy has undergone a major merging event in the past. Other studies have found that the GC populations have approximately isotropic orbits (Zepf et al. 2000; Côté et al. 2001, 2003; Bridges et al. 2006; Schuberth et al. 2009). However, separating the blue and red GCs have shown there may be slight anisotropy as well, with M87 (Côté et al. 2001) and NGC 4649 (Bridges et al. 2006; Hwang et al. 2008) as examples. In the case of NGC 5128 studied here, we therefore suggest that the PNe may be following more radial orbits than the GCs.

\section{DISCUSSION}

The kinematics of the GCSs that have been performed in other giant elliptical galaxies based on current samples of > 100 GCs includes: NGC 1407 (Romanowksy et al. 2009), an elliptical galaxy in the Eridanus A group at a distance of $20.9 \mathrm{Mpc}$ (Forbes et al. 2006); NGC 4649 (M60) (Bridges et al. 2006; Hwang et al. 2008), a luminous giant elliptical galaxy in the Virgo cluster of galaxies at a distance of $17.3 \mathrm{Mpc}$ (Mei et al. 2007): NGC 4486 (M87) (Kissler-Patig \& Gebhardt 1998; Cohen 2000; Côté et al. 2001), a supermassive cD galaxy, located at a distance of $16.7 \pm 0.2 \mathrm{Mpc}$ (Mei et al. 2007) in the Virgo cluster of galaxies; NGC 4472 (M49) (Sharples et al. 1998; Zepf et al. 2000; Côté et al. 2003), a giant elliptical galaxy and also the brightest member of the Virgo cluster of galaxies, located at a distance of $16.4 \pm 0.2$ Mpc (Mei et al. 2007); NGC 4636 (Schuberth et al. 2006; Lee et al. 2010), the southernmost giant elliptical galaxy from the dynamical center of the Virgo cluster of galaxies, located 14.7 Mpc away (Tonry et al. 2001); and NGC 1399, a giant elliptical galaxy and also the brightest galaxy in the Fornax cluster of galaxies, $19 \mathrm{Mpc}$ away (Richtler et al. 2004; Schuberth et al. 2009). The main findings of these studies have been well summarized in Hwang et al. (2008) and Romanowksy et al. (2009) and we do not repeat these here.

We find that the kinematic trends of NGC 1407 (Romanowksy et al. 2009) are quite similar to NGC 5128 found in this study. Both systems have larger rotation in the metal-rich GCs (at least in the inner-most region where both datasets have strong azimuthal coverage), with the rotation of the metal-rich GCs around the isophotal major axis. Both systems have velocity dispersions that appear flat or decreasing in the inner regions with a potential increase at larger radii. They are also both dominated by dispersion over their entire GC population. Both NGC 5128 and NGC 1407 are the only two elliptical galaxies in a group environment with large samples of GCs available in order to perform a kinematic analysis. With a sample of 2 galaxies, it is a far stretch to propose that the GCSs in elliptical galaxies found in group environments have similar kinematics due to similar formation histories. With this in mind, if these galaxies hierarchically built up the majority of their stellar content at early times, later small accretions and minor merging will not severely disrupt the orbital and kinematic properties in the outer halo. The kinematics from the major episodes of star formation within these galaxies may still be intact.

\subsection{Implications for Galaxy Formation}

The monolithic collapse scenario (Eggen et al. 1962; Tinslev 1972; Larson 1974, 1975; Silk 1977; Arimoto \& Yoshii 1987) suggests the GCs in massive galaxies should be old, forming at high redshift. The collapse would be very rapid indicating that the spread in old ages should be small and the kinematic properties of the GCs should be quite similar. Any rotation that exists in the GCs would come from tidal torques from nearby forming galaxies (Peebles 1969). The expected amount of rotation would be small, as the angular momentum of the initial cloud would be small to begin with in order to form the spheroidal shape of the elliptical galaxy. This scenario is not supported by the trend towards younger ages as metallicity increases in the GCS (Peng et al. 2004b; Beasley et al. 2008; Woodley et al. 2010). Neither is it supported by the large amounts of rotation that are seen for some GCSs (for the metal-rich GCs in NGC 4636, and both metal-rich and metal-poor GCs in NGC 4486 and NGC 4649) or by the different rotational properties of some metal-rich and metal-poor GCs (in NGC 4636, NGC 4486, and NGC 4472, for example).

The galaxy merging model (Schweizer 1987; Ashman \& Zepf 1992) would naturally suggest younger ages of the metal-rich GCs, which form, according to this scenario, only in the merging events, while the metal-poor GCs pre-exist in the progenitor galaxies. This theory expects a multimodal color distribution of the GCS. Numerical simulations by Bekki et al. (2002) have shown that the metal-poor GCs in this scenario are spatially extended while the newly formed metal-rich GCs are more centrally concentrated and more extended along the isophotal major axis of the galaxy. While the above properties are not disputed here, this does not explain the old ages for the majority of metal-rich GCs found in previous work on NGC 5128, unless all the major merging events took place at very early times ( 10 Gyr ago). Any existing angular momentum would be transferred to the outer regions of the remnant galaxy from the merging process (Bekki et al. 2005). The more extended metal-poor GC population should have a higher rotation signature than the metal-rich GCs, particularly in the outer regions. We do not see this in the GCs of NGC 5128 or in the GCs of the other giant elliptical galaxies studied, with the exception of NGC 4472, which generally agrees with the galaxy merger model.

A hierarchical merging scenario, combining the multiphase dissipational collapse model (Forbes et al. 1997) and accretion scenarios (Côté et al. 1998, 2000, 2002), proposed by Beasley et al. (2002, 2003) and 
Strader et al. (2005), is more consistent with our findings for NGC 5128. This naturally explains the GC color bimodality and also predicts old ages for both metalrich and metal-poor GCs (with the metal-poor GCs having slightly older ages). The old ages obtained for the stellar population in the halo of NGC 5128, as well as similar chemical enrichment between the metal-rich GCs and stellar halo (Rejkuba et al. 2005; Harris 2008), are consistent with this scenario as well. This hierarchical scenario also supports the younger metal-rich GCs in NGC 5128 forming in either more recent merging events and/or on a more extended timescale. In the sparse environment of NGC 5128, the formation of the more metal-rich GCs could be extended because the density in the group environment is lower than that in clusters of galaxies. This is supported by both the range of ages for the metal-rich GCs as well as the $[\alpha / \mathrm{Fe}]$ values suggesting longer formation timescales (Woodley et al. 2010), compared to GCs in other giant galaxies. In a typical massive collapse, the metal-poor GCs would not necessarily be expected to form with large amounts of rotation and instead they should have a large velocity dispersion (Hwang et al. 2008). However, with the accretion of small protogalaxies in the early Universe, the GCs may have some rotation as well as orbital anisotropy. The metal-rich GCs, in this formation scenario, can vary in rotation, depending on the degree of the second collapse (Forbes et al. 1997). We see evidence for this in NGC 5128 in both the old ages and kinematic results.

Bekki et al. (2005) have numerically simulated the kinematic signatures of GCs from the merging of Milky Way-type disk galaxies with pre-existing old metal-poor and metal-rich GCs. To simplify their simulations, they assumed that the initial GCSs in the progenitor galaxies were supported by dispersion with no rotation. However, we see rotation in the GCSs of M31 (Perrett et al. 2002) and the Milky Way (Harris 2001). They also assumed the mergers were dissipationless, but we do see some small fraction of young GCs in NGC 5128. However, these numerical simulations allow us to compare the kinematic properties that we see in the GCS of NGC 5128 to the generally expected kinematics from two old merging spiral galaxies. Their results indicate that both the metal-rich and metal-poor populations would have increasing rotational signatures with galactocentric radius. Bekki et al. (2005) also find the velocity dispersions of the GCSs are flat or declining out to large radii caused by their radial anisotropic orbits. Lastly, they found a variety of kinematic alignments for the metal-rich and metal-poor GCs.

In our kinematic study of NGC 5128, we do not see a strong case for significant rotation in either metallicity subpopulation, and also no significant increase with radius in the rotation signature. We do, however, see indications for flat and declining velocity dispersions in the inner $20^{\prime}$ of NGC 5128 for both metal-rich and metalpoor GCs, and a different axis of rotation for the metalrich and metal-poor GCs. The results found here are not clearly consistent with the idea of the GCs forming their kinematic signature from a major disk-disk merger, though this kind of origin is not ruled out. In the case of other galaxies, NGC 1399 shows little to no rotation in either metal-rich or metal-poor GCs and NGC 1407 also show little to moderate rotation signatures as well.
There is an important implication from the study of Bekki et al. (2005) that should not be underplayed. Their results suggest that the initial kinematic signatures of GCs in the progenitor galaxies undergo orbital mixing and it may, therefore, not be possible to trace the original kinematics of the GCs (Hwang et al. 2008). It may only be possible to examine the GC kinematics from the most recent major interaction. As suggested by Kissler-Patig et al. (1998), it would then be very difficult to use the current kinematic signature to trace the orbital history of GCs. In this respect, we can suggest that the most recent major interaction in NGC 5128 was not likely a major disk-disk merger. Again, it seems more likely that the galaxy formed hierarchically in early times, with many infalling protogalaxies.

\section{CONCLUSIONS}

We have obtained spectroscopy with LDSS2/Magellan, VIMOS/VLT, and Hydra/CTIO in order to measure the radial velocities of GCs in the nearby elliptical galaxy, NGC 5128. With these datasets, we have remeasured radial velocities for 218 known GCs as well as confirmed 155 new GCs. There are now 605 GCs confirmed in NGC 5128 and 564 of these GCs have measured radial velocities. This is the second largest kinematic dataset of GCs in any galaxy after NGC 1399 (Schuberth et al. 2009).

The large sample of GCs with radial velocity measurements allows us to perform a new kinematical analysis of the system. We have investigated the systemic velocity, the rotation amplitude, the rotation axis, and the velocity dispersion for the entire GCS, as well as the metal-rich and metal-poor GC subpopulations. We have also analyzed these kinematic properties as a function of galactocentric radius. We find the metal-poor GCs have a low rotation $26 \pm 15 \mathrm{~km} \mathrm{~s}^{-1}$ extending out to $45^{\prime}$, and does not have a well-defined rotation axis. The motion of the metal-poor GCs is supported by dispersion with a measured value of $149 \pm 4 \mathrm{~km} \mathrm{~s}^{-1}$. It has a flat/declining velocity dispersion profile extending to $20^{\prime}$, at which point it appears to increase. The metal-rich GCs have small to moderate rotation of $43 \pm 15 \mathrm{~km} \mathrm{~s}^{-1}$ out to $40^{\prime}$ around the isophotal major axis of the galaxy. It has similar velocity dispersion for the whole system $\left(156 \pm 4 \mathrm{~km} \mathrm{~s}^{-1}\right)$ compared to the metal-poor GCs. The metal-rich velocity dispersion profile decreases out to a galactocentric radius of $5^{\prime}$ and then flattens. This increase in the velocity dispersion profiles in the metal-poor GC subpopulation may be a result of the incomplete and spatially biased sample beyond $15^{\prime}$ in the GCS, or caused by Milky Way halo contamination. Or perhaps it may be due to the presence of a substructure among the GCS which could artificially inflat the velocity dispersion in the outer halo, or it could also be an indicator of an extensive dark matter halo. As a consequence of the low rotation amplitude, both the metal-poor and metal-rich subpopulations have low values of the rotation parameter of $0.17 \pm 0.09$ and $0.28 \pm 0.10$, respectively.

We have also analyzed the kinematics for the GCs that have estimated ages and metallicities from the work of Woodley et al. (2010) and found that the young $(<5$ Gyr) metal-rich GCs rotate on a different axis than the entire metal-rich GC population. If these GCs were formed from the accretion of a gas-rich satellite galaxy, 
we could expect their kinematic signature to be different from the older GCs.

We estimate the mass and mass-to-light ratio of NGC 5128 using its GCs. We find a mass of $(5.9 \pm 2.0) \times$ $10^{11} \mathrm{M}_{\odot}$ out to $20^{\prime}$ with $\mathrm{M} / \mathrm{L}_{B}=16.4 \mathrm{M}_{\odot} / \mathrm{L}_{B \odot}$. Our mass estimates determined using the metal-rich GCs and the metal-poor GCs all agree within uncertainties. This mass estimate clearly puts NGC 5128 in the class of giant elliptical galaxies, with a potentially large dark matter halo.

K.A.W., W.E.H, and G.L.H.H. thank NSERC for their financial support. M.G. thanks Proyecto DI-36-09/R for financial support. D.G. gratefully acknowledges support from the Chilean Centro de Astrofísica FONDAP No. 15010003 and the Chilean Centro de Excelencia de Astrofísica y Technologías Afines (CATA). K.A.W. also thanks Dr. Peter Frinchaboy and Dr. Matthew Walker for helpful discussions regarding data reduction of the Hydra/CTIO dataset.

\section{REFERENCES}

Abadi, M. G., Navarro, J. F., \& Steinmetz, M. 2006, MNRAS, 365,747

Arimoto, N. \& Yoshii, Y. 1987, A\&A, 173, 23

Armandroff, T. E. 1989, AJ, 95, 375

Ashman, K. M. \& Zepf, S. E. 1992, ApJ, 384, 50

Ashman, K. M. \& Zepf, S. E. 1998, Globular Cluster Systems (Cambridge, MA: Cambridge University Press)

Bahcall, J. \& Tremaine, S. 1981, ApJ, 244, 805

Baugh, C. M., Cole, S., Frenk, C. S., \& Lacey, C. G. 1998, ApJ, 498, 504

Beasley, M. A., Baugh, C. M., Frobes, D. A., Sharples, R. M., \& Frenk, C. S. 2002, MNRAS, 333, 383

Beasley, M. A., Harris, W. E., Harris, G. L. H., \& Forbes, D. A. 2003, MNRAS, 340, 341

Beasley, M. A., Bridges, T., Peng, E. W., Harris, W. E., Harris, G. L. H., Forbes, D. A., \& Mackie, G. 2008, MNRAS, 386, 1443

Bekki, K., Bekki, K., Forbes, D. A., Beasley, M. A., \& Couch, W. J. 2002, MNRAS, 335, 1176

Bekki, K., Forbes, D. A., Beasley, M. A., \& Couch, W. J. 2003, MNRAS, 344, 1334

Bekki, K., Beasley, M. A., Brodie, J. P., \& Forbes, D. A. 2005, MNRAS, 363, 1211

Bergond, G., Zepf, S. E., Romanowsky, A. J., Sharples, R. M., \& Rhode, K. L. 2006, A\&A, 448, 155

Bridges, T. J., Gebhardt, K., Sharples, R., Faifer, F. R., Forte, J. C., Beasley, M. A., Zepf, S. E., Forbes, D. A., Hanes, D. A., \& Pierce, M. 2006, MNRAS, 373, 157

Bridges, T. J., Rhode, K. L., Zepf, S. E., \& Freeman, K. C. 2007, ApJ, 658, 980

Brodie, J. P. \& Huchra, J. P. 1990, ApJ, 362, 503

Burstein, D., Faber, S. M., Gaskell, C. M., \& Krumm, N. 1984, ApJ, 287, 586

Carlson, M. N., Holtzman, J. A., Watson, A. M., Grillmair, C. J., Mould, J. R., Ballester, G. E., Burrows, C. T., Clarke, J. T., Crisp, D., Evans, R. W., Gallagher, J. S. III, Griffiths, R. E., Hester, J. J., Hoessel, J. G., Scowen, P. A., Stapelfeldt, K. R. Trauger, J. T., \& Westphal, J. A. 1998, AJ, 115, 1778

Chien, L. -H., Barnes, J. E., Kewley, L. J., \& Chambers, K. C., 2007, ApJ, 660, 105

Cohen, J. G. 2000, AJ, 119, 162

Cohen, J. G., Blakeslee, J. P., \& Côté, P. 2003, ApJ, 592, 866

Cole, S., Lacey, C. G., Baugh, C. M., \& Frenk, C. S. 2000, MNRAS, 319, 168

Côté, P., Marzke, R. O., \& West, M. J. 1998, ApJ, 501, 554

Côté, P., Marzke, R. O., West, M. J., \& Minniti, D. 2000, ApJ, 533,869

Côté, P., McLaughlin, D.E., Hanes, D.A., Bridges, T.J., Geisler, D., Merritt, D., Hesser, J.E., Harris, G.L.H., \& Lee, M.G. 2001, ApJ, 559, 828

Côté, P., West, M. J., \& Marzke, R. O. 2002, ApJ, 567, 853

Côté, P., McLaughlin, D. E., Cohen, J. G., \& Blakeslee, J. P. 2003, ApJ, 591, 850

Dekel, A. \& Woo, J. 2003, MNRAS, 344, 1131

Dekel, A., Stoehr, F., Mamon, G. A., Cox, T. J., Novak, G. S., \& Primak, J. R. 2005, Nature, 437, 701

Diemand, J., Madau, P., \& Moore, B. 2005, MNRAS, 364, 367

Dirsch, B., Richtler, T., Geisler, D., Forte, J., Bassion, L., \& Gieren, W. 2003, AJ, 124, 1908
Doherty, M., Arnaboldi, M., Das, P., Gerhard, O., Aguerri, J. A. L., Ciardullo, R., Feldmeier, J. J., Freeman, K. C., Jacoby, G. H., \& Murante, G. 2009, A\&A, 502, 771

Douglas, N. G., Napolitano, N. R., Romanowsky, A. J., Coccato, L., Kuijken, K., Merrifield, M. R., Arnaboldi, M., Gerhard, O., Freeman, K. C., Merrett, H. R., Noordermeer, E., \& Capaccioli, M. 2007, ApJ, 664, 257

Dufour, R. J., van den Bergh, S., Harvel, C. A., Martins, D. H., Schiffer, F. H., Talbot, R. J., Talent, D. L., \& Wells, D. C. 1979, AJ, 84, 284

Eggen, O. J., Lynden-Bell, D., \& Sandage, A. R. 1962, ApJ, 136, 748

Elson, R. A. W. \& Santiago, B. X. 1996, MNRAS, 280, 971

Elson, R. A. W. \& Walterbos, R. A. M. 1988, ApJ, 333, 594

Evans, N.W., Wilkinson, M.I., Perrett, K.M., \& Bridges, T.J. 2003, ApJ, 583, 752

Forbes, D. A., Brodie, J. P., \& Grillmair, C. J. 1997, AJ, 113, 1652

Forbes, D. A., Grillmair, C. J., Williger, G. M., Elson, R. A. W. \& Brodie, J. P. 1998, MNRAS, 293, 325

Forbes, D. A., Beasley, M. A., Brodie, J. P., \& Kissler-Patig, M. 2001, ApJ, 563, L143

Forbes, D. A., Faifer, F. R., Forte, J. C., Bridges, T. J., Beasley, M. A., Gebhardt, K., Hanes, D. A., Sharples, R., \& Zepf, S. E. 2004, MNRAS, 355,608

Forbes, D. A., Sánchez-Blázquez, P., Phan, A. T. T., Brodie, J. P., Strader, J., \& Spitler, L. 2006, MNRAS, 366, 1230

Gerhard, O., Kronawitter, A., Saglia, R. P., \& Bender, R. 2001, AJ, 121,1936

Geisler, D., Lee, M. G., \& Kim, E. 1996, AJ, 111, 1529

Gnedin, O. Y. \& Prieto, J. L. 2006, arXiv:astro-ph/0606169

Gómez, M., Geisler, D., Harris, W. E., Richtler, T., Harris, G. L. H., \& Woodley, K. A. 2006, A\&A, 447, 877

Gómez, M. \& Woodley, K. A. 2007, ApJ, 670, 105

Groudfrooij, P., Schweizer, F., Gilmore, D., \& Whitmore, B. C. 2007, AJ, 133, 2737

Graham, J. A. 1998, ApJ, 502, 245

Harris, G. L. H., Geisler, D., Harris, W. E., Schmidt, B. P., Hesser, J. E., Reid, M. A., Milne, M., Hulme, S. C. \& Kidd, T. T. 2004a, AJ, 128, 712

Harris, G. L. H., Harris, W. E., \& Geisler, D. 2004b, AJ, 128, 723

Harris, W. E. 1991, ARA\&A, 29, 543

Harris, G. L. H., Geisler, D., Harris, H. C., \& Hesser, J. E. AJ, 104,613

Harris, W. E. 1996, AJ, 112, 1487

Harris, W. E. Star Clusters: Saas-Fee Advanced Course, Springer-Verlag: New York

Harris, W. E. \& Harris, G. L. H. 2002, AJ, 123, 3108

Harris, W. E., Harris, G. L. H., Barmby, P., McLaughlin, D. E.. \& Forbes, D. A., 2006, AJ, 132, 2187

Harris, W. E. 2008, IN MPA/ESO/MPE/USM Joint Astronomy Conference, Chemical Evolution of Dwarf Galaxies and Stellar Clusters

Harris, G. L. H., Rejkuba, M. \& Harris, W. E. 2009, submitted to PASA

Heisler, J., Tremaine, S., \& Bahcall, J. N. 1985, ApJ, 298, 8

Hempel, M., Hilker, M., Kissler-Patig, M., Puzia, T. H., Minniti, D., \& Goudrooij, P. 2003, A\&A, 405, 487

Hesser, J. E., Harris, H. C., van den Bergh, S., \& Harris, G. L. H. 1984, ApJ, 276, 491 
Hesser, J. E., Harris, H. C., Harris, G. L. H. ApJ, 303, L51

Holtzman, J. A., Faber, S. M., Shaya, E. J., Lauer, T. R., Groth, J., Hunter, P. A., Baum, W. A., Ewald, S. P., Hester, J. J., Light, R. M., Lynds, C. R., O’Neil, E. J. Jr., \& Westphal, J. A 1992, AJ, 103,691

Humphrey, P. J., Buote, D. A., Gastaldello, F., Zappacosta, L., Bullock, J. S., Brighenti, F., \& Mathews, W. G. 2006, ApJ, 649,899

Hwang, H. S., Lee, M. G., Park, H. S., Kim, S. C., Park, J.-H., Sohn, Y.-J., Lee, S.-G., Rey, S.-C., Lee, Y.-W., \& Kim, H.-I. 2008, ApJ, 674, 869

Karachentsev, I. D.,Sharina, M. E., Dolphin, A. E., Grebel, E. K., Geisler, D., Guhathakurta, P., Hodge, P. W., Karachentseva, V. E., Sarajedini, A., \& Seitzer, P. 2002, A\&A, 385, 21

Kauffmann, G., White, S. D., \& Guiderdone, B. 1993, MNRAS, 264, 201

Kauffmann, G., Heckman, T. M., White, S. D. M., Charlot, S., Tremonti, C., Peng, E. W., Seibert, M., Brinkmann, J., Nichol, R. C., SubbaRao, M., \& York, D. 2003, MNRAS, 341, 54

King, I. 1962, AJ, 67, 471

Kissler-Patig, M., Brodie, J. P., Schroder, L. L., Forbes, D. A., Grillmair, C. J., \& Huchra, J. P. 1998, AJ, 115, 105

Kissler-Patig, M. \& Gebhardt, K. 1998, AJ, 116, 2237

Kronawitter, A., Saglia, R. P., Gerhard, O., \& Bender, R. 2000, A\&AS, 144, 53

Kundu, A., Zepf, S. E., Hempel, M., Morton, D., Ashman, K. M., Maccarone, T. J., Kissler-Patig, M., Puzia, T. H., \& Vesperini, E. 2005, ApJ, 634, L41

Kundu, A. \& Zepf, S. E. 2007, ApJ, 660, L109

Kundu, A. \& Whitmore, B. C. 2001, AJ, 121, 2950

Larsen, S. S. 1999, A\&AS, 139, 393

Larsen, S. S., Brodie, J. P., Huchra, J. P., Forbes, D. A., \& Grillmair, C. J. 2001, AJ, 121, 2974

Larson, R. B. 1974, MNRAS, 166, 585

Larson, R. B. 1975, MNRAS, 173, 671

Lee, M. G., Park, H. S., Hwang, H. S., Arimoto, N., Tamura, N., \& Onodera, M. 2010, ApJ, 709, 1083

Maíz Apellániz, J. \& Úbeda, L. 2005, ApJ, 629, 873

Malin, D. F. 1978, Nature, 276, 591

Mamon, G. A. \& xxokas, E. L. 2005, MNRAS, 362, 95

Mathews, W. G. \& Brighenti, F. 2003, ARA\&A, 41, 191

McLaughlin, D. E., Barmby, P., Harris, W. E., Forbes, D. A., \& Harris, G. L. H. 2008, MNRAS, 384, 563

McLaughlin, D. E. 1999, ApJ, 512, L9

Mei, S., Blakeslee, J. P., Côté, P., Tonry, J. L., West, M. J., Ferrarese, L., Jordán, A., Peng, E. W., Anthony, A., \& Merritt, D. 2007, ApJ, 655,144

Méndez, R. H., Teodorescu, A. M., Kudritzki, R.-P., \& Burkert, A. 2009, ApJ, 691, 228

Merrett, H. R., Kuijken, K., Merrifield, M. R., Romanowsky, A. J., Douglas, N. G., Napolitano, N. R., Arnaboldi, M., Capaccioli, M., Freeman, K. C., Gerhard, O., Evans, N. W., Wilkinson, M. I., Halliday, D., Bridges, T. J., \& Carter, D. 2003, MNRAS, 346, L62

Miller, B. W., Whitmore, B. C., Schweizer, F., \& Fall, S. M. 1997, AJ, 114, 2381

Napolitano, N. R., Romanowsky, A. J., Coccato, L., Capaccioli, M., Douglas, N. G., Noordermeer, E., Gerhard, O., Arnaboldi, M., de Lorenzi, F., Kuijken, K., Merrifield, M. R., O'Sullivan, E., Cortesi, A., Das, P., \&Freeman, K. C. 2009, MNRAS, 393, 329

Peebles, P. J. E. 1969, ApJ, 155, 393

Peebles, P. J. E. 1980, The Large-Scale Structure of the Universe (Princeton: Princeton University Press)

Peng, E. W., Ford, H., C., Freeman, K. C. \& White, R. L. 2002, AJ, 124,3144

Peng, E. W., Ford, H. C., \& Freeman, K. C. 2004a, ApJ, 602, 685

Peng, E. W., Ford, H. C., \& Freeman, K. C. 2004b, ApJ, 602, 705

Peng, E. W., Ford, H. C., \& Freeman, K. C. 2004c, ApJS, 150, 367

Perrett, K. M., Bridges, T. J., Hanes, D. A., Irwin, M. J., Brodie, J. P., Carter, D., Huchra, J. P., \& Watson, F. G. 2002, AJ, 123, 2490

Pierce, M., Beasley, M. A., Forbes, D. A., Bridges, T. J., Gebhardt, K., Faifer, F. R., Forte, J. C., Zepf, S. E., Sharples, R., Hanes, D. A., \& Proctor, R. 2006, MNRAS, 366, 1253
Pryor, C. \& Meylan, G. 1993, in ASP Conf. Ser. 50: Structure and Dynamics of Globular Clusters, 357

Puzia, T. H., Zepf, S. E., Kissler-Patig, M., Hilker, M., Minniti, D., \& Goudfrooij, P. 2002, A\&A, 391, 453

Puzia, T. H., Kissler-Patig, M., Thomas, D., Maraston, C., Saglia, R. P., Bender, R., Goudfrooij, P., \& Hempel, M. 2005, A\&A, 439, 997

Puzia, T. H., Kissler-Patig, M. \& Goudfrooij, P., 2006, ApJ, 648, 383

Puzia, T. H., \& Sharina, M. E. 2008, ApJ, 674, 909

Reed, L. G., Harris, G. L. H., \& Harris, W. E. 1994, AJ, 107, 555

Rejkuba, M., Minniti, D., Silva, D. R., \& Bedding, T. R. 2001 A\&A, 379, 781

Rejkuba, M., Minniti, D., Courbin, F., \& Silva, D. R. 2002, ApJ, 564,688

Rejkuba, M., Greggio, L., Harris, W. E., Harris, G. L. H., \& Peng, E. W. 2005, ApJ, 631, 262

Rejkuba, M., Dubath, P., Minniti, D., \& Meylan, G. 2007, A\&A, 469, 147

Rhode, K. \& Zepf, S. E. 2001, AJ, 121, 210

Richtler, T., Dirsch, B., Gebhardt, K., Geisler, D., Hilker, M., Alonso, M. V., Forte, J. C., Grebel, E. K., Infante, L., Larsen, S., Minniti, D., \& Rejkuba, M. 2004, AJ, 127, 2094

Romanowsky, A., Douglas, L. G., Arnaboldi, M., Kuijken, K., Merrifield, M. R., Napolitano, N. R., Capaccioli, M., \& Freeman, K. C. 2003, Science, 301, 1696

Romanowsky, A. J., Strader, J., Spitler, L. R., Johnson, R. Brodie, J. P., Forbes, D. A., \& Ponman, T. 2009, AJ, 137, 4956 Santos, M. R. 2003, In Extragalactic Globular Cluster Systems, Ed. M. Kissler-Patig, pg. 348

Schiminovich, D., van Gorkum, J. H., van der Hulst, J. M., \& Kasow, S. 1994, ApJ, 423, L101

Schuberth, Y., Richtler, T., Dirsch, B., Hilker, M., Larsen, S. S., Kissler-Patig, M., \& Mebold, U. 2006, A\&A, 459, 391

Schuberth, Y., Richtler, T., Hilker, M., Dirsch, B., Bassion, L. P., Romanowsky, A. J., \& Infante, L. 2009, arXiv:0911.0420

Schweizer, F. 1987, In Nearly Normal Galaxies. From the Planck Time to the Present, Ed. S. M. Faber, pg. 18

Schweizer, F. \& Seitzer, P. 1993, ApJ, 417, L29

Schweizer, F., Miller, B. W., Whitmore, B. C., \& Fall, S. M. 1996, AJ, 112,1839

Schweizer, F. \& Seitzer, P. 1998, AJ, 116, 2206

Sharina, M. E., Afanasiev, V. L., \& Puzia, T. H. 2006, MNRAS, 372,1259

Sharples, R. M., Zepf, S. E., Bridges, T. J., Hanes, D. A., Carter, D., Ashman, K. M., Geisler, D., 1998, AJ, 115, 2337

Silk, J. 1977, ApJ, 214, 152

Somerville, R. S., Primack, J. R., \& Faber, S. M. 2001, MNRAS, 320,504

Strader, J., Brodie, J. P., Cenarro, A. J., Beasley, M. A., \& Forbes, D. A. 2005, AJ, 130, 1315

Strader, J., Beasley, M. A., \& Brodie, J. P. 2007, AJ, 133, 2015

Tamura, N., Sharples, R. M., Arimoto, N., Onodera, M., Ohta, K., \& Yamada, Y. 2006, MNRAS, 373, 601

Thomas, D., Maraston, C., \& Bender, R. 2003, MNRAS, 339, 897

Thomas, D., Maraston, C., \& Korn, A. 2004, MNRAS, 351, L19

Tinsley, B. M. 1972, ApJ, 178, 319

Tonry, J. L., Dressler, A., Blakeslee, J. P., Ajhar, E. A., Fletcher, A. B., Luppino, G. A., Metzger, M. R., \& Moore, C. B. 2001, ApJ, 546, 681

Toomre, A. 1977, The Evolution of Galaxies and Stellar Populations, ed. B. Tinsley \& R. B. Larson (New Haven: Yale University Obs.), 401

Trager, S. C., Worthey, G., Faber, S. M., Burstein, D., \& Gonzalez, J. J. 1998, ApJS, 116, 1

Trancho, G., Bastian, N., Miller, B. W., \& Schweizer, F. 2007, ApJ, 664, 284

van Dokkum, P. 2001, PASP, 113, 1420

van den Bergh, S., Hesser, J. E., \& Harris, G. L. H. 1981, AJ, 86, 24

White, S. D. \& Frenk, C. S. 1991, ApJ, 379, 52

White, S. D. \& Rees, M. J. 1978, MNRAS, 183, 341

Whitmore, B. C., Schweizer, F., Leitherer, C., Borne, K., Robert, C. 1993, AJ, 106, 1354

Whitmore, B. C. \& Schweizer, F. 1995, AJ, 109, 960

Whitmore, B. C., Zhang, Q., Leitherer, C., Fall, S. M., Schweizer, F., \& Miller, B. W. 1999, AJ, 118, 1551 
Woodley, K. A., Harris, W. E. \& Harris, G. L. H. 2005, AJ, 129, 2654

Woodley, K. A. 2006, AJ, 132, 2424

Woodley, K. A., Harris, W. E., Beasley, M. A., Peng, E. W., Bridges, T. J., Forbes, D. A., \& Harris, G. L. H. 2007, AJ, 134, 494

Woodley, K. A., Harris, W. E., Puzia, T. H., Gómez, M., Harris, G. L. H., \& Geisler, D. 2009, 2010, ApJ, 708, 1335

Worthey, G. \& Ottaviani, D. L. 1997, ApJS, 111, 377
Worthey, G., Faber, S. M., Gonzalez, J. J., \& Burstein, D. 1994, ApJS, 94, 687

Zepf, S. E., Carter, D., Sharples, R. M., \& Ashman, K. 1995, ApJ, 445, L19

Zepf, S. E., Ashman, K. M., English, J., Freeman, K. C., \& Sharples, R. M. 1999, AJ, 118, 752

Zepf, S. E., Beasley, M. A., Bridges, T. J., Hanes, D. A., Sharples, R. M., Ashman, K. M., \& Geisler, D. 2000, AJ, 120, 2928

Zinn, R. 1985, ApJ, 293, 424

Zinn, R. 1990, J.R. Astron. Soc. Can., 84, 89 
Kinematics of Globular Clusters in NGC 5128

TABLE 1

The Observation Data Summary

\begin{tabular}{lllll}
\hline \hline Instrument & R.A. (J2000) & Decl. (J2000) & Time (hr) \\
& & & & \\
\hline LDSS-2 & 132618.31 & -424917.5 & 1.0 \\
LDSS-2 & 132610.00 & -4253 & 40.0 & 0.7 \\
LDSS-2 & 132610.00 & -425720.0 & 0.7 \\
LDSS-2 & 132535.37 & -425127.6 & 0.7 \\
LDSS-2 & 132537.00 & -425615.0 & 0.5 \\
LDSS-2 & 132537.00 & -430900.0 & 0.6 \\
LDSS-2 & 132516.00 & -431415.0 & 1.0 \\
LDSS-2 & 132510.00 & -431830.0 & 1.0 \\
LDSS-2 & 132444.00 & -425100.0 & 0.8 \\
LDSS-2 & 132444.00 & -425330.0 & 1.0 \\
LDSS-2 & 132457.00 & -430330.0 & 0.7 \\
LDSS-2 & 132449.00 & -430800.0 & 0.7 \\
LDSS-2 & 132449.00 & -431300.0 & 1.0 \\
VIMOS & 132546.03 & -424443.7 & 1.1 \\
VIMOS & 132617.05 & -430030.4 & 1.7 \\
VIMOS & 132439.04 & -425959.8 & 1.1 \\
VIMOS & 132643.61 & -431552.0 & 3.3 \\
VIMOS & 132431.02 & -43 & 1536.7 & 1.7 \\
Hydra & 132516.91 & -425808.0 & 4.5 \\
Hydra & 132533.82 & -4302 & 49.6 & 4.5 \\
Hydra & 132458.60 & -425825.7 & 3.5 \\
& & & & \\
\hline
\end{tabular}


TABLE 2

Radial Velocity Measurements of Previously Confirmed Globular Clusters in NGC 5128

\begin{tabular}{|c|c|c|c|c|c|c|}
\hline GC ID & $\begin{array}{c}\text { R.A. } \\
\text { (J2000) }\end{array}$ & $\begin{array}{c}\text { Decl. } \\
\text { (J2000) }\end{array}$ & $\begin{array}{c}\mathrm{v}_{r, L D S S-2} \\
\left(\mathrm{~km} \mathrm{~s}^{-1}\right)\end{array}$ & $\begin{array}{c}\mathrm{v}_{r, V I M O S} \\
\left(\mathrm{~km} \mathrm{~s}^{-1}\right)\end{array}$ & $\begin{array}{l}\mathrm{v}_{r, H y d r a} \\
\left(\mathrm{~km} \mathrm{~s}^{-1}\right)\end{array}$ & $\begin{array}{c}\mathrm{v}_{r} \\
\left(\mathrm{~km} \mathrm{~s}^{-1}\right)\end{array}$ \\
\hline GC0001 & 132501.16 & -425651.5 & - & $503 \pm 16$ & $546 \pm 39$ & $516 \pm 10$ \\
\hline GC0005 & 132344.19 & $-4311 \quad 11.8$ & - & $712 \pm 32$ & - & $642 \pm 1$ \\
\hline GC0007 & 132354.52 & -432001.1 & - & $273 \pm 52$ & - & $274 \pm 49$ \\
\hline GC0009 & 132358.58 & $-4257 \quad 17.0$ & - & $305 \pm 38$ & - & $590 \pm 144$ \\
\hline GC0010 & 132358.76 & -430135.2 & - & $482 \pm 31$ & - & $496 \pm 21$ \\
\hline GC0011 & 132359.51 & $\begin{array}{lll}-43 & 17 & 29.1\end{array}$ & - & $617 \pm 24$ & - & $652 \pm 37$ \\
\hline GC0018 & 132410.97 & $\begin{array}{lll}-43 & 12 & 52.8\end{array}$ & - & $496 \pm 17$ & - & $439 \pm 118$ \\
\hline GC0020 & 132418.92 & -431430.1 & - & $686 \pm 21$ & - & $749 \pm 32$ \\
\hline GC0021 & 132421.40 & -430236.8 & - & $604 \pm 21$ & - & $596 \pm 17$ \\
\hline GC0022 & 132423.72 & $\begin{array}{lll}-43 & 07 & 52.1\end{array}$ & - & $599 \pm 20$ & - & $613 \pm 16$ \\
\hline GC0023 & 132423.98 & -425410.7 & - & $606 \pm 33$ & - & $582 \pm 81$ \\
\hline GC0024 & 132424.15 & -425420.6 & - & $574 \pm 14$ & - & $616 \pm 25$ \\
\hline GC0028 & 132428.44 & -425752.9 & - & $516 \pm 50$ & - & $558 \pm 97$ \\
\hline GC0031 & 132429.73 & -430206.5 & - & $559 \pm 19$ & - & $595 \pm 202$ \\
\hline GC0032 & 132431.35 & $\begin{array}{llll}-43 & 11 & 26.7\end{array}$ & $775 \pm 34$ & - & - & $734 \pm 25$ \\
\hline GC0033 & 132432.17 & -431056.9 & $813 \pm 40$ & - & - & $775 \pm 32$ \\
\hline GC0037 & 132436.87 & $\begin{array}{lll}-43 & 19 & 16.2\end{array}$ & - & $505 \pm 49$ & - & $570 \pm 31$ \\
\hline GC0038 & 132437.75 & $\begin{array}{lll}-43 & 16 & 26.5\end{array}$ & - & - & $213 \pm 10$ & $212 \pm 10$ \\
\hline GC0040 & 132438.98 & $\begin{array}{llll}-43 & 20 & 06.4\end{array}$ & - & $355 \pm 20$ & - & $363 \pm 1$ \\
\hline GC0041 & 132440.39 & $\begin{array}{lll}-43 & 18 & 05.3\end{array}$ & - & $754 \pm 18$ & $597 \pm 16$ & $725 \pm 1$ \\
\hline GC0044 & 132440.60 & $\begin{array}{lll}-43 & 13 & 18.1\end{array}$ & - & $711 \pm 24$ & - & $699 \pm 17$ \\
\hline GC0046 & 132441.20 & -430145.6 & $483 \pm 49$ & - & - & $518 \pm 18$ \\
\hline GC0048 & 132443.60 & -42537.3 & $550 \pm 48^{\mathrm{a}}$ & - & - & $509 \pm 15$ \\
\hline GC0050 & 132444.58 & $\begin{array}{lll}-43 & 02 & 47.3\end{array}$ & $731 \pm 44$ & - & - & $718 \pm 16$ \\
\hline GC0053 & 132445.78 & $\begin{array}{lll}-43 & 02 & 24.5\end{array}$ & $444 \pm 58$ & $558 \pm 24$ & - & $503 \pm 17$ \\
\hline GC0054 & 132446.46 & $\begin{array}{llll}-4 & 04 & 11.6\end{array}$ & $736 \pm 58$ & - & - & $712 \pm 20$ \\
\hline GC0056 & 132447.10 & $\begin{array}{lll}-43 & 06 & 01.7\end{array}$ & $550 \pm 53$ & - & - & $528 \pm 12$ \\
\hline GC0059 & 132447.61 & -431048.5 & $343 \pm 39$ & - & - & $344 \pm 37$ \\
\hline GC0060 & 132448.06 & $\begin{array}{lll}-4 & 08 & 14.2\end{array}$ & $804 \pm 46$ & - & - & $786 \pm 18$ \\
\hline GC0061 & 132448.71 & -425235.5 & $512 \pm 172$ & - & $509 \pm 63$ & $509 \pm 59$ \\
\hline GC0062 & 132448.97 & -425748.4 & - & $625 \pm 28$ & - & $611 \pm 17$ \\
\hline GC0063 & 132449.38 & $\begin{array}{lll}-43 & 08 & 17.7\end{array}$ & $474 \pm 226$ & - & - & $567 \pm 76$ \\
\hline GC0064 & 132450.09 & $-4307 \quad 36.2$ & $588 \pm 41$ & - & - & $594 \pm 36$ \\
\hline GC0066 & 132450.87 & -430122.9 & - & - & $546 \pm 18^{\mathrm{b}}$ & $550 \pm 10$ \\
\hline GC0067 & 132451.49 & $\begin{array}{llll}-43 & 12 & 11.1\end{array}$ & $640 \pm 30$ & $616 \pm 20$ & - & $624 \pm 13$ \\
\hline GC0068 & 132452.06 & $\begin{array}{lll}-43 & 04 & 32.7\end{array}$ & - & - & $158 \pm 90$ & $191 \pm 27$ \\
\hline GC0070 & 132453.29 & -430434.8 & $271 \pm 77$ & - & - & $485 \pm 23$ \\
\hline GC0072 & 132454.18 & -425450.4 & $540 \pm 46$ & $534 \pm 32$ & - & $564 \pm 33$ \\
\hline GC0074 & 132454.35 & -425324.8 & - & $712 \pm 14$ & $739 \pm 15$ & $745 \pm 7$ \\
\hline GC0075 & 132454.49 & -430534.7 & - & $733 \pm 20$ & - & $695 \pm 45$ \\
\hline GC0076 & 132454.55 & -424858.7 & $397 \pm 48$ & - & - & $439 \pm 26$ \\
\hline GC0077 & 132454.73 & $\begin{array}{lll}-43 & 01 & 21.7\end{array}$ & - & $783 \pm 18$ & $765 \pm 14^{\mathrm{c}}$ & $791 \pm 1$ \\
\hline GC0081 & 132455.71 & -432039.1 & - & $295 \pm 16$ & - & $279 \pm 38$ \\
\hline GC0082 & 132456.06 & -425429.6 & $513 \pm 54$ & $459 \pm 17$ & $535 \pm 30$ & $529 \pm 26$ \\
\hline GC0083 & 132456.08 & $\begin{array}{lll}-43 & 10 & 16.4\end{array}$ & $692 \pm 33^{\mathrm{d}}$ & $606 \pm 20$ & - & $687 \pm 31$ \\
\hline GC0086 & 132457.44 & -430108.1 & $613 \pm 55$ & - & - & $685 \pm 9$ \\
\hline GC0091 & 132458.21 & -425610.0 & - & $528 \pm 13$ & - & $561 \pm 1$ \\
\hline GC0095 & 132459.92 & $\begin{array}{l}-430908.6 \\
\end{array}$ & - & $374 \pm 34$ & - & $374 \pm 34$ \\
\hline GC0098 & 132500.64 & $-4305 \quad 30.3$ & $468 \pm 64$ & - & - & $503 \pm 21$ \\
\hline GC0099 & 132500.83 & $-4311 \quad 10.6$ & $941 \pm 66$ & $919 \pm 38$ & - & $941 \pm 66$ \\
\hline GC0103 & 13251.60 & -425440.9 & $686 \pm 60$ & & - & $603 \pm 32$ \\
\hline GC0107 & 132501.86 & $\begin{array}{lll}-42 & 52 & 27.8\end{array}$ & $635 \pm 73$ & $401 \pm 47$ & - & $635 \pm 73$ \\
\hline GC0108 & 132502.76 & $\begin{array}{lll}-43 & 11 & 21.2\end{array}$ & $481 \pm 45$ & $388 \pm 21$ & - & $456 \pm 26$ \\
\hline GC0109 & 132503.13 & -425625.1 & - & $502 \pm 13$ & - & $523 \pm 8$ \\
\hline GC0110 & 132503.18 & $\begin{array}{lll}-43 & 03 & 02.5\end{array}$ & - & $606 \pm 16$ & $583 \pm 22$ & $577 \pm 5$ \\
\hline GC0111 & 132503.24 & -425740.5 & - & $679 \pm 22$ & - & $648 \pm 29$ \\
\hline GC0113 & $\begin{array}{lll}13 & 25 & 03.37\end{array}$ & -425046.2 & - & $722 \pm 26$ & - & $718 \pm 10$ \\
\hline GC0115 & 132503.67 & -425121.7 & - & $384 \pm 102$ & $426 \pm 34$ & $425 \pm 34$ \\
\hline GC0117 & $\begin{array}{lll}13 & 25 & 04.48\end{array}$ & -431048.4 & - & $648 \pm 24$ & - & $626 \pm 22$ \\
\hline GC0119 & 132504.81 & -430938.8 & $520 \pm 27^{\mathrm{e}}$ & $463 \pm 13$ & - & $481 \pm 10$ \\
\hline GC0120 & 132505.02 & $\begin{array}{llll}-42 & 57 & 15.0\end{array}$ & - & $674 \pm 9$ & - & $677 \pm 7$ \\
\hline GC0121 & 132505.29 & -425805.8 & - & $807 \pm 18$ & - & $787 \pm 19$ \\
\hline GC0122 & 132505.46 & -431402.6 & $707 \pm 48$ & $680 \pm 16$ & - & $679 \pm 13$ \\
\hline GC0123 & 132505.72 & -431030.7 & $565 \pm 49$ & $430 \pm 8$ & $447 \pm 13^{\mathrm{f}}$ & $439 \pm 1$ \\
\hline GC0125 & 132506.25 & -431511.6 & - & & $588 \pm 18^{\mathrm{g}}$ & $597 \pm 10$ \\
\hline GC0126 & 132507.33 & -430829.6 & - & $532 \pm 30$ & - & $550 \pm 28$ \\
\hline GC0128 & $\begin{array}{lll}13 & 25 & 07.48\end{array}$ & $-43 \quad 1229.4$ & $503 \pm 68$ & $616 \pm 59$ & & $548 \pm 21$ \\
\hline GC0129 & 132507.62 & $\begin{array}{llll}-43 & 01 & 15.2\end{array}$ & - & $701 \pm 15$ & - & $689 \pm 11$ \\
\hline GC0130 & 132508.51 & -430257.4 & $326 \pm 50$ & $384 \pm 14$ & - & $361 \pm 12$ \\
\hline GC0135 & 132509.54 & $-42 \quad 55 \quad 18.5$ & - & $405 \pm 31$ & - & $448 \pm 23$ \\
\hline GC0137 & $1325 \quad 10.25$ & $-42 \quad 5509.5$ & - & $547 \pm 11$ & - & $576 \pm 12$ \\
\hline GC0138 & $\begin{array}{lll}13 & 25 & 10.27\end{array}$ & -425333.1 & - & $414 \pm 9$ & - & $416 \pm 8$ \\
\hline GC0143 & 132511.17 & -430309.6 & $395 \pm 67$ & $447 \pm 49$ & - & $426 \pm 21$ \\
\hline GC0144 & 132511.98 & $\begin{array}{llll}-43 & 04 & 19.3\end{array}$ & $865 \pm 77$ & $612 \pm 11$ & - & $622 \pm 18$ \\
\hline GC0145 & 132512.11 & $\begin{array}{llll}-42 & 57 & 25.2\end{array}$ & - & $487 \pm 12$ & - & $490 \pm 10$ \\
\hline GC0146 & 132512.21 & -431633.9 & $510 \pm 25^{\mathrm{h}}$ & - & - & $573 \pm 16$ \\
\hline GC0148 & 132512.45 & $\begin{array}{llll}-43 & 14 & 07.4\end{array}$ & - & $467 \pm 24$ & - & $526 \pm 33$ \\
\hline GC0149 & 132512.84 & -425659.8 & - & $586 \pm 29$ & - & $664 \pm 141$ \\
\hline
\end{tabular}


Kinematics of Globular Clusters in NGC 5128

TABLE 3

Radial Velocity Measurements of Newly Confirmed Globular Clusters in NGC 5128

\begin{tabular}{|c|c|c|c|c|c|c|c|c|c|c|c|c|}
\hline GC ID & $\begin{array}{l}\text { R.A. } \\
\text { (J2000) }\end{array}$ & $\begin{array}{c}\text { Decl. } \\
\text { (J2000) }\end{array}$ & $\begin{array}{c}\mathrm{C} \\
(\mathrm{mag})\end{array}$ & $\begin{array}{c}\sigma_{C} \\
(\mathrm{mag})\end{array}$ & $\begin{array}{c}\mathrm{M} \\
(\mathrm{mag})\end{array}$ & $\begin{array}{c}\sigma_{M} \\
(\mathrm{mag})\end{array}$ & $\begin{array}{c}\mathrm{T}_{1} \\
(\mathrm{mag})\end{array}$ & $\begin{array}{c}\sigma_{T_{1}} \\
(\mathrm{mag})\end{array}$ & $\begin{array}{c}\mathrm{v}_{r, L D S S}-2 \\
\left(\mathrm{~km} \mathrm{~s}^{-1}\right)\end{array}$ & $\begin{array}{c}\mathrm{v}_{r, V I M O S} \\
\left(\mathrm{~km} \mathrm{~s}^{-1}\right)\end{array}$ & $\begin{array}{l}\mathrm{v}_{r, H y d r a} \\
\left(\mathrm{~km} \mathrm{~s}^{-1}\right)\end{array}$ & $\left(\begin{array}{c}\mathrm{v}_{r} \\
\left(\mathrm{~km} \mathrm{~s}^{-1}\right)\end{array}\right.$ \\
\hline GC0416 & 132433.63 & -431201.6 & 21.69 & 0.02 & 20.94 & 0.01 & 20.12 & 0.01 & $597 \pm 52$ & - & $543 \pm 10$ & $546 \pm 9$ \\
\hline GC0417 & 132442.39 & -425149.1 & 20.50 & 0.02 & 19.82 & 0.02 & 19.09 & 0.01 & $681 \pm 35$ & - & $577 \pm 39$ & $634 \pm 26$ \\
\hline GC0418 & 132444.77 & -430633.3 & 22.01 & 0.06 & 20.86 & 0.05 & 19.87 & 0.03 & $487 \pm 102$ & - & $363 \pm 32$ & $374 \pm 30$ \\
\hline GC0419 & 132455.31 & $-43 \quad 1039.3$ & 21.87 & 0.02 & 20.83 & 0.02 & 19.89 & 0.01 & $653 \pm 57$ & - & $658 \pm 32$ & $656 \pm 27$ \\
\hline GC0420 & 132455.97 & $\begin{array}{lll}-43 & 02 & 15.9\end{array}$ & 21.29 & 0.03 & 20.64 & 0.02 & 19.87 & 0.03 & $807 \pm 65$ & $763 \pm 40$ & $526 \pm 87$ & $735 \pm 28$ \\
\hline GC0421 & 132459.58 & -430640.8 & 22.62 & 0.03 & 21.49 & 0.03 & 20.47 & 0.01 & $841 \pm 124$ & $719 \pm 31$ & - & $726 \pm 30$ \\
\hline GC0422 & 132503.28 & $\begin{array}{llll}-43 & 08 & 14.4\end{array}$ & 21.11 & 0.03 & 20.56 & 0.02 & 19.76 & 0.01 & $666 \pm 140$ & $646 \pm 38$ & $805 \pm 60$ & $690 \pm 31$ \\
\hline GC0423 & 132504.28 & $-4317 \quad 10.8$ & 22.36 & 0.04 & 21.80 & 0.03 & 20.92 & 0.02 & $577 \pm 73$ & $499 \pm 51$ & & $524 \pm 41$ \\
\hline GC0424 & 132506.87 & $\begin{array}{lll}-43 & 02 & 40.2\end{array}$ & 20.65 & 0.01 & 19.93 & 0.01 & 19.13 & 0.01 & $247 \pm 49$ & $367 \pm 18$ & $410 \pm 38$ & $354 \pm 14$ \\
\hline GC0425 & 132509.60 & -430437.9 & 21.65 & 0.02 & 20.94 & 0.01 & 20.11 & 0.01 & $658 \pm 34$ & $657 \pm 32$ & - & $648 \pm 19$ \\
\hline GC0426 & $13 \quad 2514.18$ & -430446.6 & 21.23 & 0.03 & 20.67 & 0.02 & 19.87 & 0.02 & $705 \pm 120$ & $881 \pm 36$ & - & $858 \pm 30$ \\
\hline GC0427 & 132521.49 & -431929.6 & 19.59 & 0.01 & 19.23 & 0.01 & 18.66 & 0.01 & $163 \pm 57$ & - & - & $163 \pm 57$ \\
\hline GC0428 & 132522.16 & -431638.8 & 21.06 & 0.04 & 20.48 & 0.04 & 19.76 & 0.02 & $401 \pm 55$ & - & $466 \pm 96$ & $417 \pm 47$ \\
\hline GC0429 & 132522.88 & $\begin{array}{llll}-43 & 08 & 10.1\end{array}$ & 20.62 & 0.01 & 19.20 & 0.01 & 18.14 & 0.01 & $146 \pm 110$ & - & - & $146 \pm 110$ \\
\hline GC0430 & 132524.38 & $-4257 \quad 16.8$ & - & - & 19.25 & 0.29 & 18.90 & 0.01 & $503 \pm 75$ & - & - & $503 \pm 75$ \\
\hline GC0431 & 132528.72 & $\begin{array}{lll}-42 & 50 & 12.3\end{array}$ & 20.60 & 0.04 & 19.93 & 0.04 & 19.36 & 0.01 & $663 \pm 54$ & $593 \pm 40$ & $687 \pm 38$ & $646 \pm 24$ \\
\hline GC0432 & 132534.08 & -431045.7 & 19.87 & 0.31 & 20.81 & 0.07 & 20.00 & 0.05 & $396 \pm 84$ & - & - & $396 \pm 84$ \\
\hline GC0433 & 132536.11 & -425616.8 & 22.61 & 0.03 & 22.58 & 0.03 & 22.00 & 0.09 & $575 \pm 56$ & - & - & $575 \pm 56$ \\
\hline GC0434 & 132537.85 & -425628.0 & 20.20 & 0.01 & 20.59 & 0.01 & 18.51 & 0.00 & $505 \pm 51$ & $478 \pm 17$ & - & $480 \pm 16$ \\
\hline GC0435 & 132538.53 & $\begin{array}{lll}-42 & 57 & 19.9\end{array}$ & 21.32 & 0.02 & 20.30 & 0.03 & 19.42 & 0.01 & $706 \pm 63$ & - & - & $706 \pm 63$ \\
\hline GC0436 & 132541.71 & -425831.6 & 20.34 & 0.02 & 19.29 & 0.01 & 18.52 & 0.01 & $523 \pm 71$ & $440 \pm 15$ & $478 \pm 18$ & $456 \pm 10$ \\
\hline GC0437 & $13 \quad 25 \quad 45.23$ & -425423.8 & 22.04 & 0.03 & 21.00 & 0.04 & 20.14 & 0.02 & $490 \pm 113$ & - & - & $490 \pm 113$ \\
\hline GC0438 & $1325 \quad 45.91$ & -425141.2 & 21.34 & 0.02 & 21.55 & 0.01 & 19.56 & 0.01 & $837 \pm 59$ & - & - & $837 \pm 59$ \\
\hline GC0439 & 132547.00 & -425529.9 & 20.56 & 0.05 & 19.75 & 0.03 & 18.89 & 0.02 & $700 \pm 48$ & - & - & $700 \pm 48$ \\
\hline GC0440 & 132550.59 & -425140.1 & 20.19 & 0.02 & 19.60 & 0.01 & 18.95 & 0.02 & $622 \pm 50$ & - & $649 \pm 42$ & $637 \pm 32$ \\
\hline GC0441 & 132554.48 & -425727.6 & 20.97 & 0.02 & 19.91 & 0.01 & 19.12 & 0.01 & $698 \pm 67$ & $597 \pm 11$ & $626 \pm 27$ & $603 \pm 10$ \\
\hline GC0442 & 132609.37 & $-4253 \quad 17.5$ & 21.09 & 0.03 & 20.38 & 0.02 & 19.67 & 0.02 & $615 \pm 81$ & - & $646 \pm 69$ & $632 \pm 52^{\mathrm{a}}$ \\
\hline GC0443 & 132612.11 & -424903.5 & 19.87 & 0.01 & 19.31 & 0.01 & 18.73 & 0.01 & $225 \pm 35$ & - & - & $225 \pm 35$ \\
\hline GC0444 & 132620.63 & -425346.0 & 21.49 & 0.01 & 20.80 & 0.01 & 20.15 & 0.01 & $271 \pm 64$ & - & - & $271 \pm 64$ \\
\hline GC0480 & 132350.49 & -431143.9 & 20.96 & 0.03 & 21.87 & 0.03 & 23.02 & 0.02 & - & $646 \pm 61$ & - & $646 \pm 61$ \\
\hline GC0481 & 132354.18 & -432156.6 & 21.89 & 0.05 & 22.81 & 0.03 & 24.10 & 0.03 & - & $512 \pm 84$ & - & $512 \pm 84$ \\
\hline GC0482 & 132403.98 & $\begin{array}{lll}-43 & 17 & 20.3\end{array}$ & 21.73 & 0.03 & 22.55 & 0.02 & 23.45 & 0.03 & - & $599 \pm 105$ & - & $599 \pm 105$ \\
\hline GC0483 & 132409.86 & -432239.0 & 20.89 & 0.05 & 21.90 & 0.03 & 23.02 & 0.03 & - & $756 \pm 37$ & - & $756 \pm 37$ \\
\hline GC0484 & 132410.98 & $-4312 \quad 15.8$ & 20.53 & 0.02 & 21.47 & 0.01 & 22.58 & 0.01 & - & $498 \pm 22$ & - & $498 \pm 22$ \\
\hline $\mathrm{GC} 0485^{\mathrm{d}}$ & 132413.76 & -430533.4 & 19.96 & 0.01 & 20.83 & 0.01 & 21.84 & 0.01 & - & $388 \pm 20$ & - & $388 \pm 20$ \\
\hline GC0486 & 132419.45 & -425431.5 & 18.98 & 0.01 & 19.83 & 0.01 & 20.77 & 0.01 & - & $566 \pm 12$ & $503 \pm 25$ & $554 \pm 11$ \\
\hline GC0487 & 132420.60 & -430807.9 & 19.88 & 0.01 & 20.83 & 0.01 & 22.01 & 0.01 & - & $579 \pm 17$ & $553 \pm 53$ & $576 \pm 16$ \\
\hline GC0488 & 132427.23 & -425725.7 & 20.99 & 0.03 & 21.92 & 0.03 & 22.87 & 0.02 & - & $559 \pm 35$ & - & $559 \pm 35$ \\
\hline GC0489 & 132441.51 & $\begin{array}{lll}-43 & 12 & 53.2\end{array}$ & 20.30 & 0.03 & 21.11 & 0.02 & 21.76 & 0.01 & - & $506 \pm 41$ & - & $506 \pm 41$ \\
\hline GC0490 & 132447.40 & -430959.8 & 20.20 & 0.01 & 21.14 & 0.01 & 22.15 & 0.01 & - & $568 \pm 22$ & - & $568 \pm 22$ \\
\hline GC0491 & 132454.98 & -431136.9 & 20.40 & 0.04 & 21.41 & 0.03 & 22.46 & 0.02 & - & $670 \pm 19$ & - & $670 \pm 19$ \\
\hline GC0492 & 132456.61 & -431223.6 & 20.74 & 0.03 & 21.42 & 0.03 & 22.00 & 0.02 & - & $713 \pm 28$ & - & $713 \pm 28$ \\
\hline GC0493 & 132457.89 & -430706.3 & 20.11 & 0.03 & 21.04 & 0.02 & 21.97 & 0.01 & - & $559 \pm 40$ & - & $559 \pm 40$ \\
\hline GC0494 & 132458.30 & -431833.6 & 20.20 & 0.02 & 20.95 & 0.02 & 21.55 & 0.01 & - & $676 \pm 42$ & - & $676 \pm 42$ \\
\hline GC0495 & 132458.96 & -431128.2 & 19.98 & 0.02 & 20.93 & 0.01 & 21.99 & 0.01 & - & $494 \pm 17$ & $619 \pm 47$ & $508 \pm 15$ \\
\hline GC0496 & 132459.80 & -425733.2 & 19.68 & 0.01 & 20.47 & 0.02 & 21.11 & 0.01 & - & $470 \pm 33$ & $635 \pm 23$ & $581 \pm 18$ \\
\hline GC0497 & 132500.89 & -432107.7 & 20.37 & 0.01 & 21.37 & 0.01 & 22.48 & 0.01 & - & $492 \pm 25$ & $437 \pm 44$ & $478 \pm 21$ \\
\hline GC0498 & 132501.36 & -430546.5 & 20.38 & 0.04 & 21.39 & 0.01 & 22.47 & 0.01 & - & $472 \pm 36$ & - & $472 \pm 36$ \\
\hline GC0499 & 132501.81 & -430348.1 & 20.43 & 0.02 & 21.39 & 0.02 & 22.49 & 0.03 & - & $600 \pm 35$ & - & $600 \pm 35$ \\
\hline GC0500 & 132503.60 & -430140.6 & 20.42 & 0.03 & 21.15 & 0.01 & 21.85 & 0.03 & - & $386 \pm 52$ & - & $386 \pm 52$ \\
\hline GC0501 & $1325 \quad 06.14$ & -424618.8 & 20.02 & 0.01 & 20.91 & 0.01 & 21.97 & 0.01 & - & $564 \pm 71$ & - & $564 \pm 71$ \\
\hline GC0502 & 132506.68 & -430858.2 & 20.98 & 0.06 & 22.11 & 0.05 & 23.28 & 0.04 & - & $240 \pm 53$ & - & $240 \pm 53$ \\
\hline GC0503 & $1325 \quad 07.36$ & -430323.9 & 19.08 & 0.01 & 20.69 & 0.01 & 21.18 & 0.01 & - & $670 \pm 41$ & - & $670 \pm 41$ \\
\hline GC0504 & 132508.94 & -430853.7 & 20.04 & 0.04 & 20.70 & 0.03 & 21.29 & 0.01 & - & $678 \pm 26$ & - & $678 \pm 26$ \\
\hline GC0505 & 132512.69 & -430155.9 & 19.80 & 0.04 & 20.54 & 0.03 & 21.57 & 0.03 & - & $578 \pm 20$ & - & $578 \pm 20$ \\
\hline GC0506 & $\begin{array}{lll}13 & 25 & 12.77\end{array}$ & -431043.5 & 20.42 & 0.04 & 21.44 & 0.04 & 22.45 & 0.02 & - & $280 \pm 29$ & - & $280 \pm 29$ \\
\hline GC0507 & 132513.04 & -425556.2 & 19.55 & 0.02 & 20.32 & 0.01 & 20.94 & 0.01 & - & $460 \pm 29$ & $478 \pm 91$ & $461 \pm 27$ \\
\hline GC0508 & 132513.70 & -424736.9 & 21.27 & 0.01 & 22.04 & 0.01 & 22.80 & 0.01 & - & $326 \pm 71$ & - & $326 \pm 71$ \\
\hline GC0509 & 132513.70 & $\begin{array}{l}-424032.4 \\
\end{array}$ & 20.73 & 0.01 & 21.45 & 0.01 & 22.22 & 0.01 & - & $306 \pm 35$ & - & $306 \pm 35$ \\
\hline GC0510 & $1325 \quad 17.68$ & -425256.5 & 20.62 & 0.05 & 21.44 & 0.06 & 22.12 & 0.02 & - & $670 \pm 45$ & - & $670 \pm 45$ \\
\hline GC0511 & $\begin{array}{lll}13 & 25 & 18.29\end{array}$ & -424752.4 & 20.81 & 0.02 & 21.66 & 0.02 & 22.62 & 0.02 & - & $487 \pm 39$ & - & $487 \pm 39$ \\
\hline GC0512 & $1325 \quad 18.92$ & -423903.6 & 19.70 & 0.02 & 20.40 & 0.02 & 21.10 & 0.01 & - & $664 \pm 34$ & $441 \pm 82$ & $631 \pm 31$ \\
\hline GC0513 & 132520.11 & -432221.8 & 20.33 & 0.01 & 21.11 & 0.01 & 21.72 & 0.01 & - & $844 \pm 54$ & - & $844 \pm 54$ \\
\hline GC0514 & 132520.74 & $\begin{array}{lll}-42 & 41 & 47.1\end{array}$ & 19.81 & 0.01 & 20.67 & 0.01 & 21.56 & 0.01 & - & $518 \pm 19$ & - & $518 \pm 19$ \\
\hline GC0515 & 132523.08 & -430620.9 & 20.65 & 0.02 & 21.12 & 0.02 & 22.11 & 0.02 & - & $591 \pm 29$ & - & $591 \pm 29$ \\
\hline GC0516 & 132523.31 & -424847.7 & 19.06 & 0.03 & 19.88 & 0.04 & 20.93 & 0.03 & - & $517 \pm 12$ & $581 \pm 28$ & $527 \pm 11$ \\
\hline GC0517 & 132524.75 & $\begin{array}{lll}-43 & 13 & 37.4\end{array}$ & 20.40 & 0.04 & 20.74 & 0.04 & 21.50 & 0.02 & - & $798 \pm 60$ & - & $798 \pm 60$ \\
\hline GC0518 & 132525.36 & -424927.5 & 21.05 & 0.03 & 21.75 & 0.03 & 22.45 & 0.03 & - & $589 \pm 63$ & - & $589 \pm 63$ \\
\hline GC0519 & 132526.09 & $-43 \quad 1122.4$ & 18.11 & 0.02 & 18.81 & 0.02 & 19.50 & 0.02 & - & $238 \pm 25$ & $250 \pm 29$ & $243 \pm 19$ \\
\hline GC0520 & 132527.18 & -425837.9 & 20.05 & 0.03 & 20.91 & 0.02 & 21.91 & 0.01 & - & $572 \pm 48$ & - & $572 \pm 48$ \\
\hline GC0521 & 132528.81 & $\begin{array}{lll}-42 & 53 & 15.9\end{array}$ & 19.82 & 0.01 & 20.80 & 0.02 & 21.60 & 0.01 & - & $160 \pm 74$ & $179 \pm 26$ & $176 \pm 24$ \\
\hline GC0522 & 132530.10 & -432233.3 & 20.44 & 0.02 & 21.33 & 0.01 & 22.26 & 0.01 & - & $573 \pm 49$ & - & $573 \pm 48$ \\
\hline GC0523b & 132533.10 & -430921.6 & 20.40 & 0.03 & 21.16 & 0.03 & 21.76 & 0.03 & - & $351 \pm 69$ & - & $351 \pm 69$ \\
\hline GC0524 & 132533.94 & -425139.4 & 20.85 & 0.02 & 22.22 & 0.01 & 22.03 & 0.02 & - & $527 \pm 61$ & - & $527 \pm 61$ \\
\hline GC0525 & 132537.17 & -424323.7 & 20.77 & 0.04 & 21.81 & 0.04 & 22.90 & 0.02 & - & $551 \pm 41$ & - & $551 \pm 41$ \\
\hline GC0526 & 132537.63 & -423932.2 & 20.37 & 0.02 & 21.11 & 0.02 & 21.93 & 0.02 & - & $507 \pm 39$ & $519 \pm 45$ & $512 \pm 29$ \\
\hline GC0527 & 132539.41 & -425824.1 & 19.84 & 0.01 & 20.44 & 0.01 & 21.08 & 0.01 & - & $262 \pm 74$ & $312 \pm 22$ & $301 \pm 19$ \\
\hline GC0528 & 132548.32 & -425506.9 & 19.91 & 0.02 & 20.61 & 0.01 & 21.60 & 0.01 & - & $489 \pm 19$ & - & $489 \pm 19$ \\
\hline CC0529 & 13255054 & -4308029 & 2030 & 002 & 2101 & 001 & 21.50 & 001 & & $720+80$ & & $720+80$ \\
\hline
\end{tabular}


TABLE 4

Gaussian Fits for the Radial Velocity Distributions

\begin{tabular}{lllll}
\hline \hline Group & \multicolumn{1}{c}{$\begin{array}{c}\mathrm{R} \\
(\text { arcmin })\end{array}$} & $\begin{array}{c}\text { mean } \\
\left(\mathrm{km} \mathrm{s}^{-1}\right)\end{array}$ & $\begin{array}{c}\text { sigma } \\
\left(\mathrm{km} \mathrm{s}^{-1}\right)\end{array}$ & $\chi_{\text {red }}^{2}$ \\
\hline All GCs & $0-5$ & $516.1 \pm 13.8$ & $149.6 \pm 10.0$ & 1.09 \\
All GCs & $5-10$ & $524.9 \pm 10.3$ & $142.6 \pm 7.4$ & 0.87 \\
All GCs & $10-15$ & $525.3 \pm 14.3$ & $152.1 \pm 10.3$ & 1.15 \\
All GCs & $15-20$ & $461.0 \pm 24.2$ & $177.7 \pm 17.8$ & 1.80 \\
All GCs & $20-45$ & $520.4 \pm 25.2$ & $207.9 \pm 18.6$ & 1.16 \\
MP GCs & $0-5$ & $493.3 \pm 21.7$ & $147.1 \pm 16.1$ & 0.88 \\
MP GCs & $5-10$ & $521.0 \pm 15.2$ & $141.4 \pm 11.0$ & 1.44 \\
MP GCs & $10-15$ & $506.3 \pm 18.8$ & $152.1 \pm 13.5$ & 1.31 \\
MP GCs & $15-20$ & $446.0 \pm 33.5$ & $169.5 \pm 24.4$ & 1.37 \\
MP GCs & $20-45$ & $475.1 \pm 31.5$ & $195.1 \pm 23.6$ & 1.42 \\
MR GCs & $0-5$ & $529.6 \pm 22.5$ & $166.8 \pm 16.6$ & 1.27 \\
MR GCs & $5-10$ & $527.3 \pm 14.1$ & $144.0 \pm 10.1$ & 0.84 \\
MR GCs & $10-15$ & $550.0 \pm 21.3$ & $148.1 \pm 15.4$ & 0.67 \\
MR GCs & $15-20$ & $474.4 \pm 34.4$ & $183.8 \pm 25.7$ & 1.33 \\
MR GCs & $20-45$ & $569.5 \pm 38.1$ & $204.1 \pm 27.7$ & 1.74 \\
& & & & \\
\hline
\end{tabular}

TABLE 5

The MASs of NGC 5128

\begin{tabular}{clllclccc}
\hline \hline GCs & $\begin{array}{c}\mathrm{R} \\
(\text { arcmin })\end{array}$ & $\mathrm{N}$ & $\begin{array}{c}v_{\text {sys }} \\
\left(\mathrm{km} \mathrm{s}^{-1}\right)\end{array}$ & $\begin{array}{c}\Omega R \\
\left(\mathrm{~km} \mathrm{~s}^{-1}\right)\end{array}$ & $\begin{array}{c}\Theta_{o} \\
(\text { deg. E of } \mathrm{N})\end{array}$ & $\begin{array}{c}\mathrm{M}_{p} \\
\left(\times 10^{11} \mathrm{M}_{\odot}\right)\end{array}$ & $\begin{array}{c}\mathrm{M}_{r} \\
\left(\times 10^{11} \mathrm{M}_{\odot}\right)\end{array}$ & $\begin{array}{c}\mathrm{M}_{t} \\
\left(\times 10^{11} \mathrm{M}_{\odot}\right)\end{array}$ \\
\hline All GCs & $0-5$ & 120 & $514 \pm 14$ & $14 \pm 21$ & $175 \pm 80$ & $1.900 \pm 0.663$ & $0.003 \pm 0.009$ & $1.902 \pm 0.663$ \\
All GCs & $0-10$ & 317 & $523 \pm 8$ & $33 \pm 13$ & $194 \pm 20$ & $4.520 \pm 1.496$ & $0.028 \pm 0.022$ & $4.548 \pm 1.496$ \\
All GCs & $0-15$ & 432 & $524 \pm 7$ & $36 \pm 11$ & $190 \pm 14$ & $6.257 \pm 1.999$ & $0.050 \pm 0.030$ & $6.307 \pm 2.000$ \\
All GCs & $0-20$ & 487 & $515 \pm 7$ & $36 \pm 11$ & $189 \pm 15$ & $9.260 \pm 2.881$ & $0.067 \pm 0.041$ & $9.327 \pm 2.881$ \\
All GCs & $0-45$ & 549 & $516 \pm 7$ & $33 \pm 11$ & $185 \pm 15$ & $17.45 \pm 4.950$ & $0.119 \pm 0.079$ & $17.56 \pm 4.949$ \\
All GCs & $5-10$ & 197 & $528 \pm 10$ & $43 \pm 16$ & $198 \pm 19$ & $2.427 \pm 0.869$ & $0.047 \pm 0.035$ & $2.472 \pm 0.869$ \\
All GCs & $5-15$ & 312 & $527 \pm 8$ & $44 \pm 13$ & $192 \pm 14$ & $4.128 \pm 1.456$ & $0.075 \pm 0.044$ & $4.203 \pm 1.457$ \\
All GCs & $5-20$ & 367 & $515 \pm 8$ & $44 \pm 13$ & $191 \pm 14$ & $5.832 \pm 2.038$ & $0.099 \pm 0.059$ & $5.931 \pm 2.038$ \\
All GCs & $5-45$ & 429 & $517 \pm 8$ & $39 \pm 12$ & $186 \pm 14$ & $10.58 \pm 3.525$ & $0.166 \pm 0.102$ & $10.75 \pm 3.527$ \\
MP GCs & $5-20$ & 180 & $508 \pm 11$ & $46 \pm 18$ & $206 \pm 18$ & $4.879 \pm 3.643$ & $0.109 \pm 0.085$ & $4.987 \pm 3.644$ \\
MP GCs & $5-45$ & 216 & $508 \pm 11$ & $31 \pm 18$ & $192 \pm 24$ & $9.942 \pm 7.153$ & $0.105 \pm 0.122$ & $10.05 \pm 7.154$ \\
MR GCs & $5-20$ & 184 & $523 \pm 11$ & $50 \pm 17$ & $174 \pm 18$ & $6.157 \pm 2.564$ & $0.127 \pm 0.086$ & $6.284 \pm 2.566$ \\
MR GCs & $5-45$ & 214 & $525 \pm 11$ & $45 \pm 16$ & $180 \pm 18$ & $9.466 \pm 3.766$ & $0.206 \pm 0.146$ & $9.672 \pm 3.769$ \\
& & & & & & & & \\
\hline
\end{tabular}

TABLE 6

The Kinematics of the Globular Cluster System of NGC 5128

\begin{tabular}{|c|c|c|c|c|c|c|c|c|c|}
\hline GCs & $\begin{array}{c}\mathrm{R} \\
(\operatorname{arcmin})\end{array}$ & $\begin{array}{c}\mathrm{R}_{a v g} \\
\text { (arcmin) }\end{array}$ & $\mathrm{N}$ & $\begin{array}{c}v_{\text {sys }} \\
\left(\mathrm{km} \mathrm{s}^{-1}\right)\end{array}$ & $\begin{array}{c}\Omega R \\
\left(\mathrm{~km} \mathrm{~s}^{-1}\right)\end{array}$ & $\begin{array}{c}\Theta_{o} \\
(\text { deg. } \mathrm{E} \text { of } \mathrm{N})\end{array}$ & $\begin{array}{c}\Theta_{o}-\Theta_{\text {major }} \\
(\text { deg. E of N) }\end{array}$ & $\begin{array}{c}\sigma_{v_{p}} \\
\left(\mathrm{~km} \mathrm{~s}^{-1}\right)\end{array}$ & $\Omega R / \sigma_{v_{p}}$ \\
\hline All GCs & $0-5$ & 3.49 & 120 & $514 \pm 14$ & $14 \pm 21$ & $175 \pm 80$ & -40 & $149 \pm 5$ & $0.09 \pm 0.14$ \\
\hline All GCs & $5-10$ & 7.31 & 197 & $528 \pm 10$ & $43 \pm 16$ & $198 \pm 19$ & -17 & $147 \pm 3$ & $0.29 \pm 0.12$ \\
\hline All GCs & $10-15$ & 11.96 & 115 & $524 \pm 14$ & $49 \pm 24$ & $184 \pm 21$ & -31 & $151 \pm 5$ & $0.32 \pm 0.17$ \\
\hline All GCs & $15-20$ & 17.32 & 55 & $446 \pm 24$ & $43 \pm 38$ & $168 \pm 43$ & -47 & $151 \pm 5$ & $0.28 \pm 0.22$ \\
\hline All GCs & $20-45$ & 26.83 & 62 & $526 \pm 22$ & $23 \pm 37$ & $55 \pm 87$ & -160 & $156 \pm 7$ & $0.15 \pm 0.25$ \\
\hline All GCs & $0-45$ & 10.82 & 549 & $517 \pm 7$ & $33 \pm 10$ & $185 \pm 15$ & -30 & $150 \pm 2$ & $0.22 \pm 0.07$ \\
\hline MP GCs & $0-5$ & 3.78 & 47 & $496 \pm 21$ & $43 \pm 29$ & $93 \pm 42$ & -122 & $144 \pm 8$ & $0.30 \pm 0.22$ \\
\hline MP GCs & $5-10$ & 7.41 & 89 & $524 \pm 16$ & $46 \pm 24$ & $228 \pm 27$ & 13 & $147 \pm 6$ & $0.31 \pm 0.17$ \\
\hline MP GCs & $10-15$ & 11.97 & 66 & $511 \pm 19$ & $47 \pm 33$ & $182 \pm 30$ & -33 & $150 \pm 7$ & $0.31 \pm 0.23$ \\
\hline MP GCs & $15-20$ & 17.48 & 25 & $426 \pm 31$ & $25 \pm 43$ & $137 \pm 114$ & -78 & $128 \pm 11$ & $0.19 \pm 0.42$ \\
\hline MP GCs & $20-45$ & 28.50 & 36 & $507 \pm 28$ & $67 \pm 50$ & $45 \pm 37$ & -170 & $171 \pm 14$ & $0.39 \pm 0.31$ \\
\hline MP GCs & $0-45$ & 11.90 & 263 & $506 \pm 9$ & $26 \pm 15$ & $175 \pm 28$ & -40 & $149 \pm 4$ & $0.17 \pm 0.09$ \\
\hline MR GCs & $0-5$ & 3.49 & 57 & $527 \pm 22$ & $65 \pm 34$ & $244 \pm 27$ & 29 & $178 \pm 11$ & $0.37 \pm 0.21$ \\
\hline MR GCs & $5-10$ & 7.23 & 107 & $527 \pm 14$ & $51 \pm 20$ & $178 \pm 22$ & -37 & $149 \pm 5$ & $0.34 \pm 0.15$ \\
\hline MR GCs & $10-15$ & 11.96 & 49 & $542 \pm 22$ & $46 \pm 36$ & $189 \pm 34$ & -26 & $148 \pm 7$ & $0.31 \pm 0.26$ \\
\hline MR GCs & $15-45$ & 20.47 & 54 & $506 \pm 24$ & $33 \pm 41$ & $168 \pm 56$ & -47 & $162 \pm 9$ & $0.20 \pm 0.21$ \\
\hline MR GCs & $0-45$ & 10.20 & 267 & $526 \pm 10$ & $43 \pm 15$ & $196 \pm 17$ & -19 & $156 \pm 4$ & $0.28 \pm 0.10$ \\
\hline
\end{tabular}


TABLE 7

The Kinematics of Globular Clusters with Ages, Metallicities, and $[\alpha / \mathrm{Fe}]^{\mathrm{a}}$

\begin{tabular}{|c|c|c|c|c|c|}
\hline Group & $\mathrm{N}$ & $\begin{array}{c}\mathrm{R}_{\text {avg }} \\
\text { (arcmin) }\end{array}$ & $\begin{array}{c}v_{\text {sys }} \\
\left(\mathrm{km} \mathrm{s}^{-1}\right)\end{array}$ & $\begin{array}{c}\Omega R \\
\left(\mathrm{~km} \mathrm{~s}^{-1}\right)\end{array}$ & $\begin{array}{c}\Theta_{o} \\
(\text { deg. } \mathrm{E} \text { of } \mathrm{N})\end{array}$ \\
\hline All GCs & 72 & 5.7 & $550 \pm 18$ & $25 \pm 26$ & $110 \pm 61$ \\
\hline GCs Age $\geq 8$ Gyr & 49 & 5.8 & $543 \pm 23$ & $27 \pm 36$ & $107 \pm 65$ \\
\hline GCs $5<\overline{\text { Age }}<8$ Gyr & 10 & 4.9 & $577 \pm 42$ & $53 \pm 78$ & $235 \pm 54$ \\
\hline GCs Age $<5$ Gyr & 13 & 5.7 & $562 \pm 51$ & $58 \pm 92$ & $80 \pm 84$ \\
\hline GCs Age $\geq 8$ Gyr \& $[\mathrm{Z} / \mathrm{H}]>-1$ & 23 & 5.9 & $574 \pm 32$ & $17 \pm 50$ & $265 \pm 143$ \\
\hline GCs Age $\geq 8$ Gyr \& $[\mathrm{Z} / \mathrm{H}] \leq-1$ & 26 & 5.7 & $515 \pm 34$ & $55 \pm 48$ & $112 \pm 51$ \\
\hline
\end{tabular}

a The ages, metallicities and $[\alpha / \mathrm{Fe}]$ used to eclassify these globular clusters are from Woodley et al. (2010). 


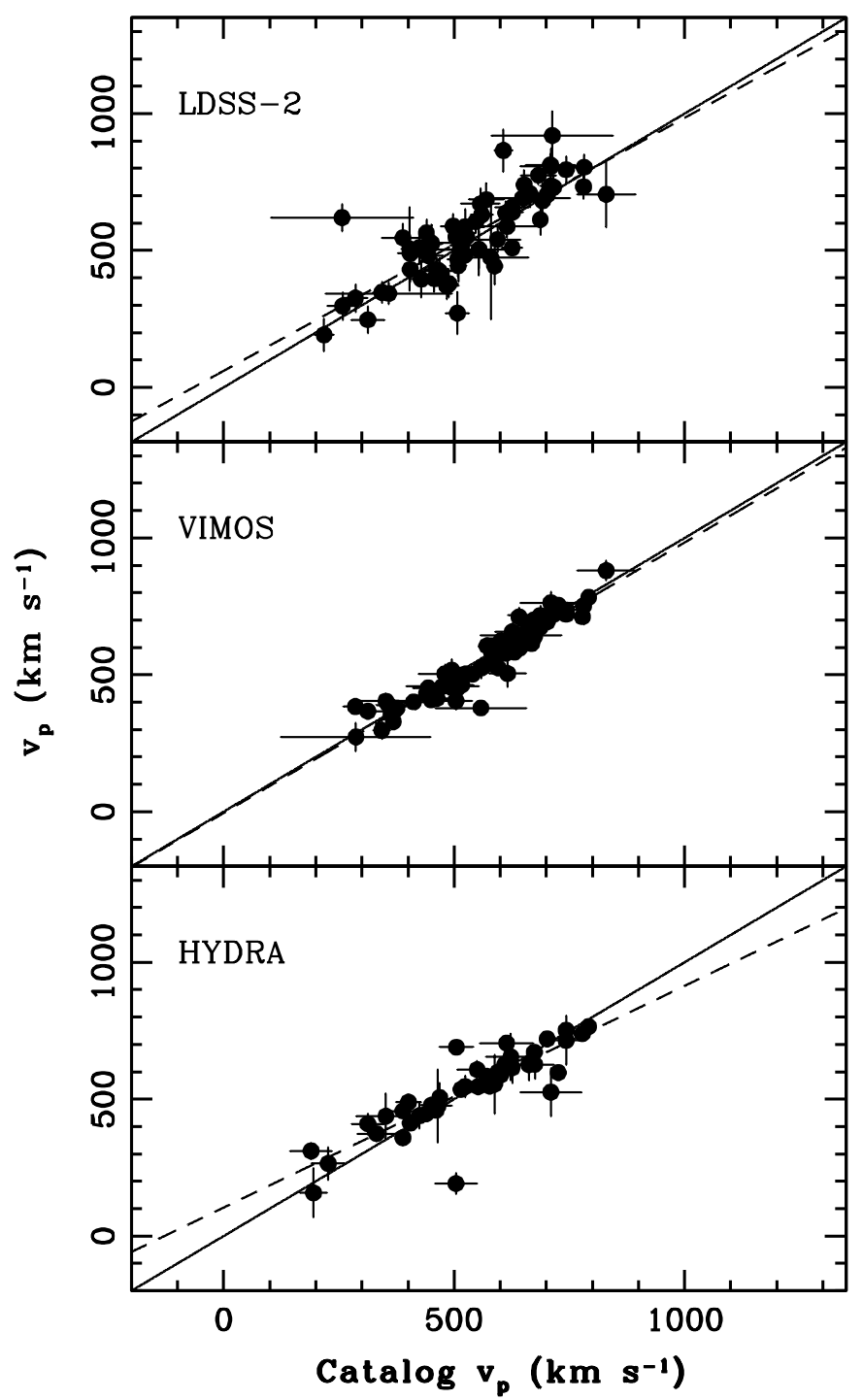

FIG. 1.- We compare radial velocities measured from our three surveys, LDSS-2 (top panel), VIMOS (middle panel), and HYDRA (bottom panel) to the weighted radial velocity measurements in the Woodley et al. (2007) catalog. The solid line is a 1:1 fit and the dashed line is the best least squares fit between the two sets of measurements. The least square fits are (slope, intercept) $=(0.92,60.2)_{L D S S-2}$, $(0.99,-4.71)_{V I M O S}$, and $\left.0.81,104.1\right)_{H Y D R A}$. We find good agreement in the LDSS-2 and VIMOS studies with the previously cataloged values. The HYDRA study has one significant outlier, which is GC0445 with cataloged velocity $504 \pm 45 \mathrm{~km} \mathrm{~s}^{-1}$ and our new measurement $192 \pm 39 \mathrm{~km} \mathrm{~s}^{-1}$. GC0445 has been measured only one previous time in Woodley et al. (2010). 


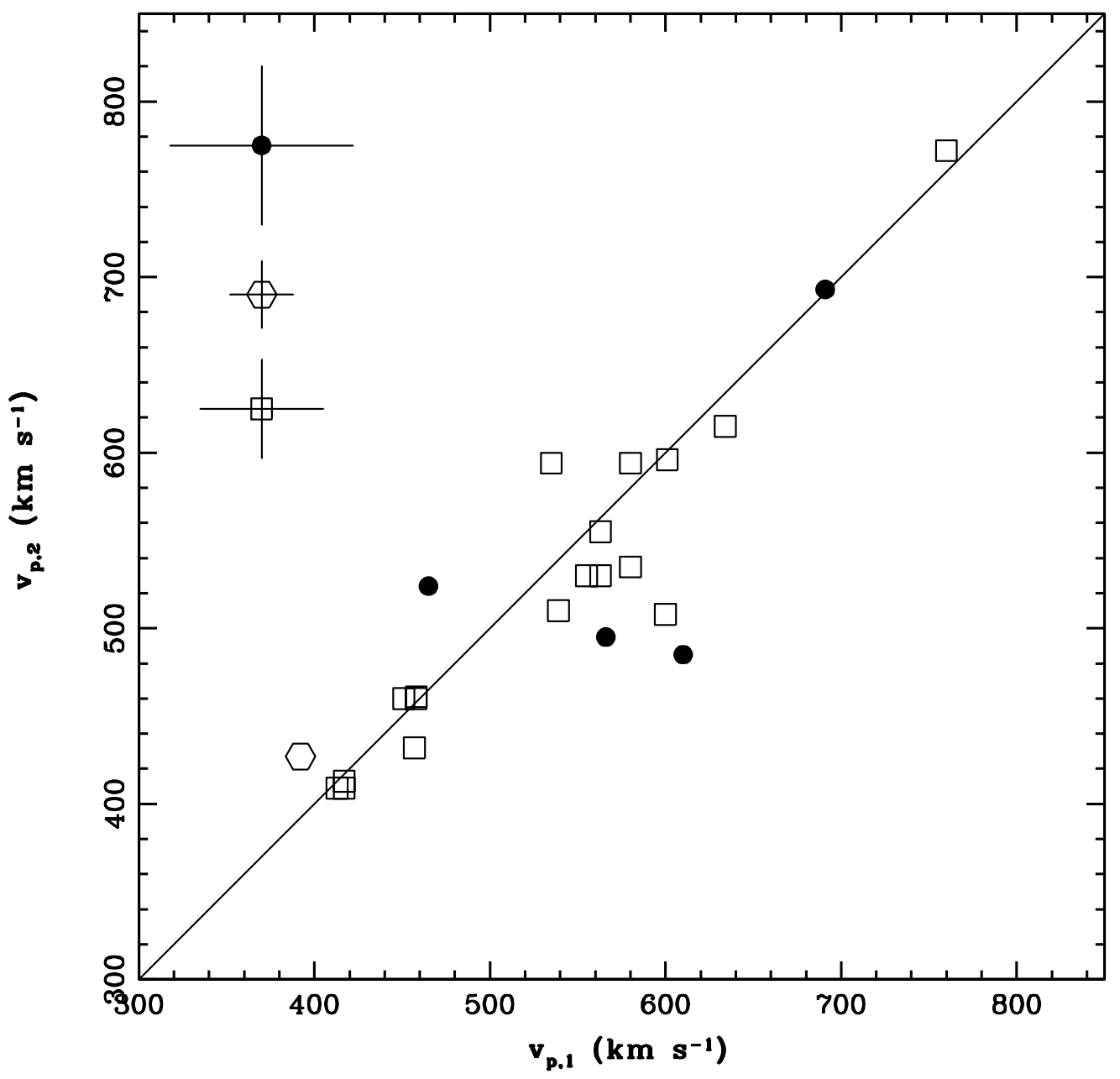

FIG. 2.- A comparison of GCs that were measured more than once within each survey. The solid line is a 1:1 fit. LDSS-2 had 4 (solid circles), VIMOS had 1 (hexagon), and HYDRA had 17 multiple measurements (squares). The least squares fit for the Hydra data has a slope of 0.96 and an intercept of 13.1, indicating decent consistency of multiple measurements within the different HYDRA fields. 


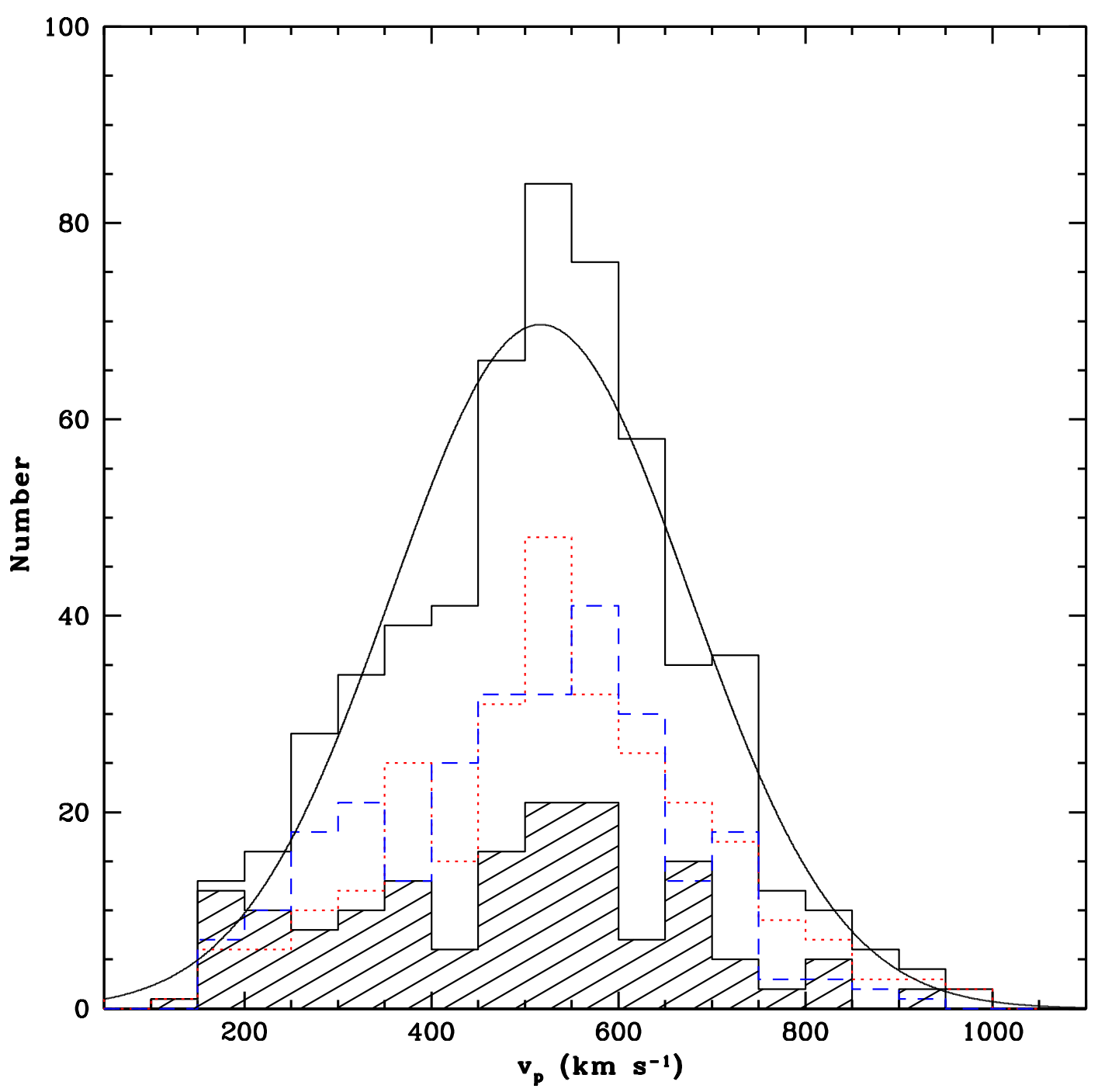

FIG. 3. - The radial velocity distribution of the entire GCS (open histogram), the metal-rich GC subpopulation (red dotted histogram), the metal-poor GC subpopulation (blue long dashed histogram), and the newly confirmed GCs from this study (hatched histogram). The entire GCS is fit with a Gaussian function using Rmix (mean $=516.7 \pm 6.8 \mathrm{~km} \mathrm{~s}^{-1}$ and sigma $=159.5 \pm 4.8 \mathrm{~km} \mathrm{~s}^{-1}$ ). See the electronic version for the color figure. 


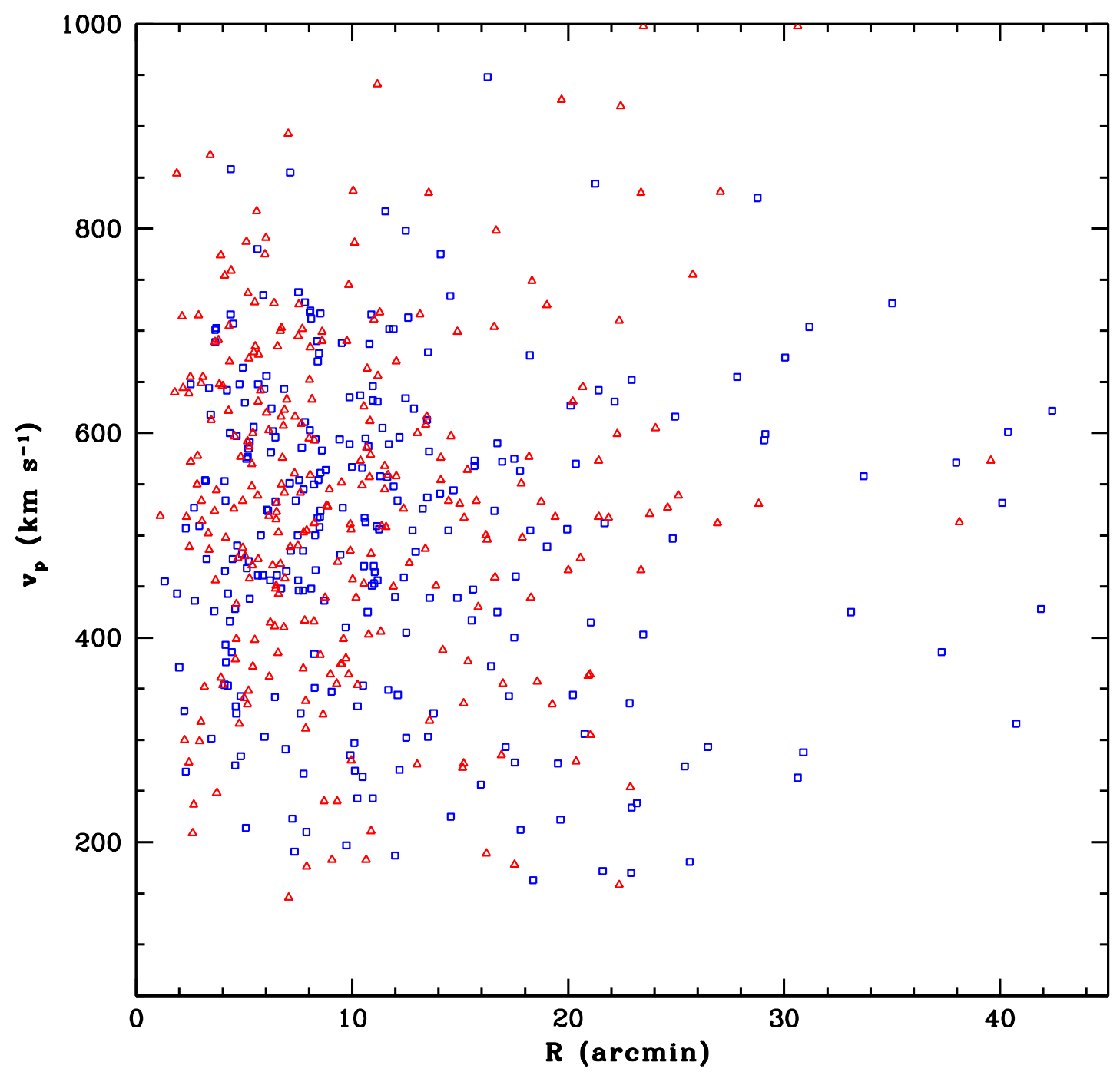

FIG. 4.- Radial velocity measurements for all metal-rich (red triangles) and metal-poor (blue squares) GCs as a function of projected galactocentric radius. See the electronic version for the color figure. 
Woodley et al.

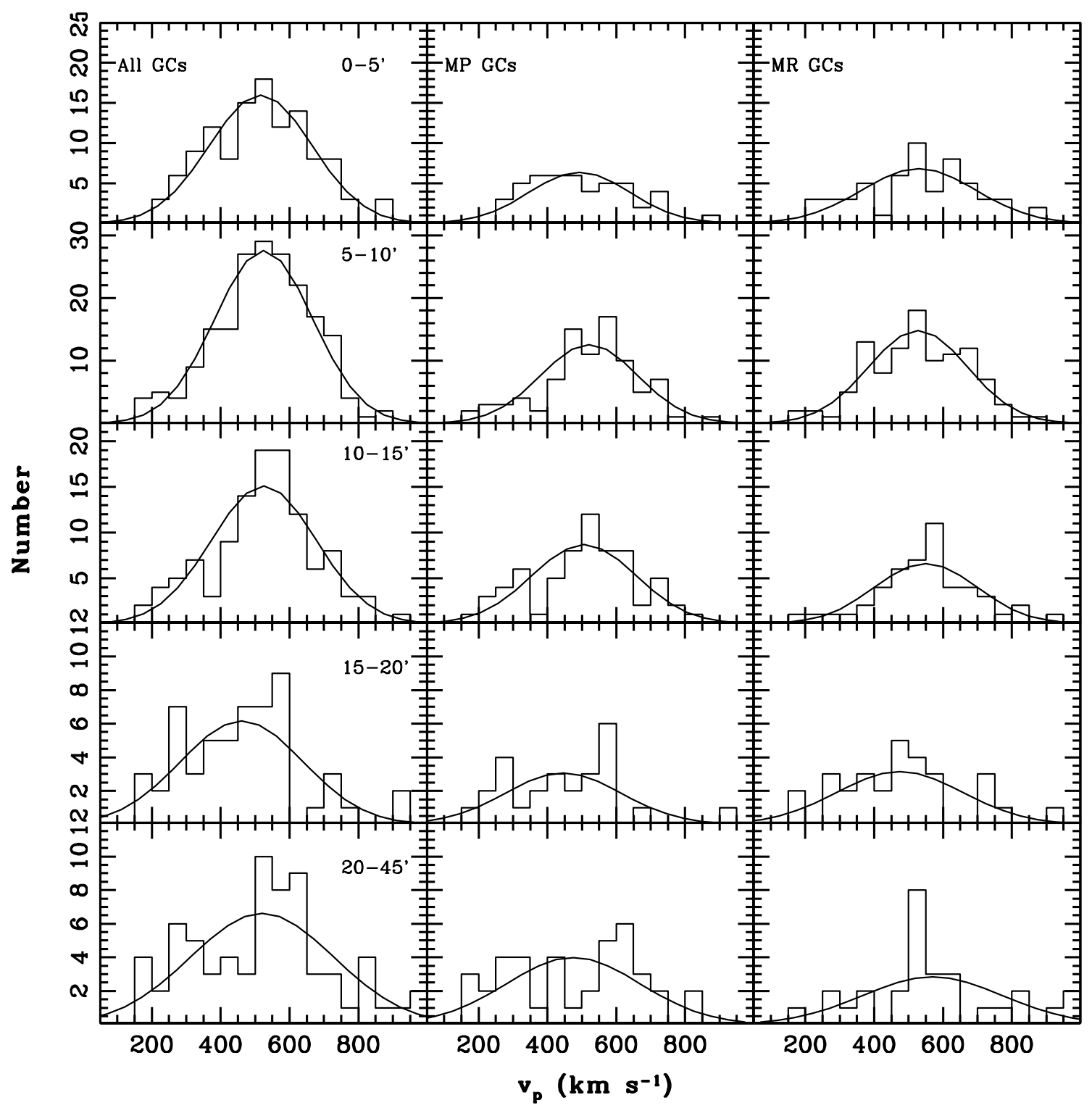

FIG. 5.- Radial velocity histograms for the entire GCS (left panels), the metal-poor (middle panels) and metal-rich (right panels) GC subpopulations. Each subpopulation is binned radially from $0^{\prime}-5^{\prime}$ (top panels), $5^{\prime}-10^{\prime}$ (2nd panels), $10^{\prime}-15^{\prime}$ (3rd panels), $15^{\prime}-20^{\prime}$ (4th panels), and $20^{\prime}-45^{\prime}$ (bottom panels). The best fits are listed in Table 4 


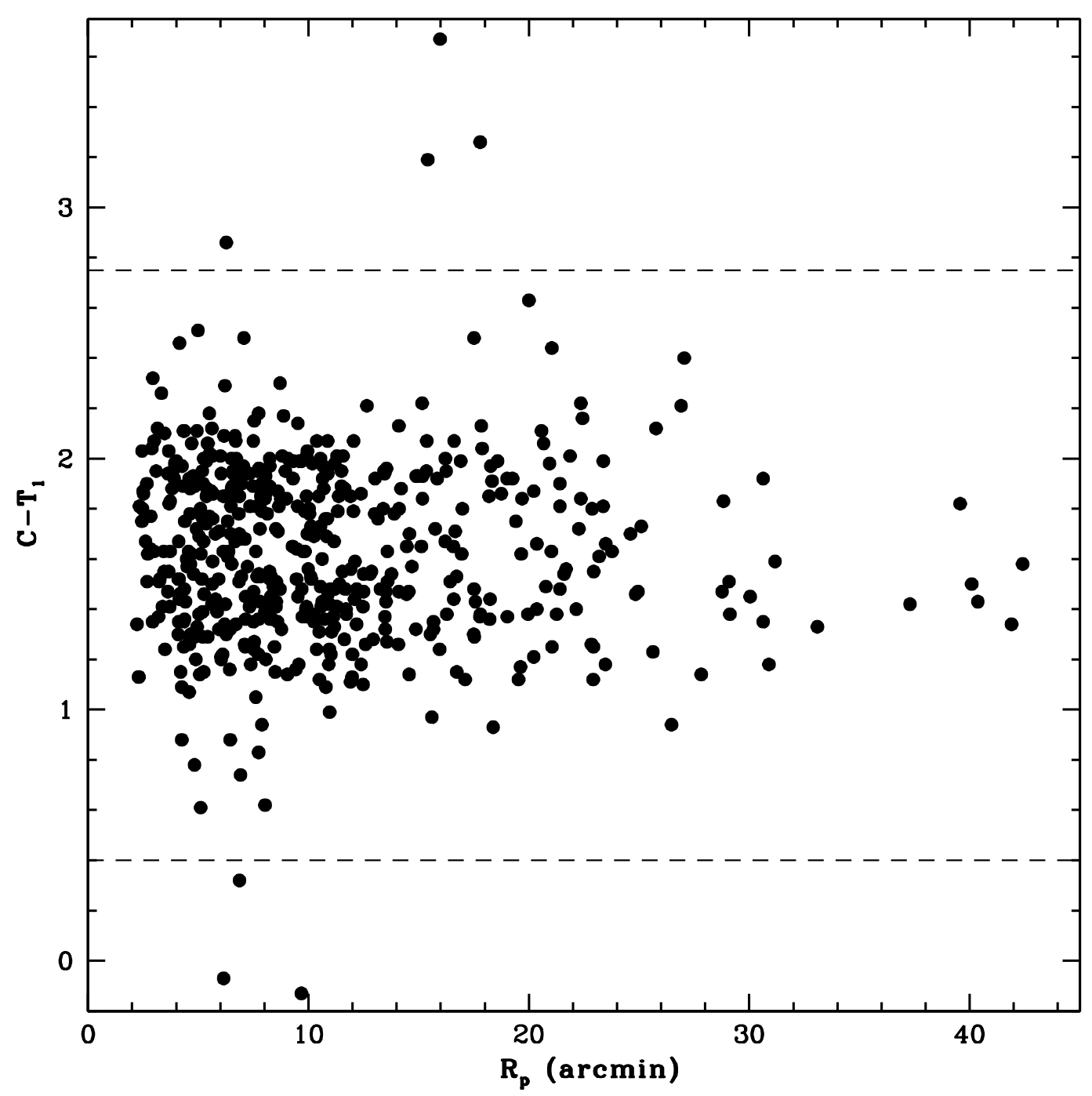

FIG. 6. - The C-T 1 color of all GCs in NGC 5128 as a function of galactocentric radius. The dashed lines indicate the bounds of the GCs included in the kinematical and subsequent dynamical analysis. Excluded are the 4 reddest (GC0078, GC0408, GC0411, and GC0552) and the 3 bluest (GC0084, GC0282, GC0432) GCs. 

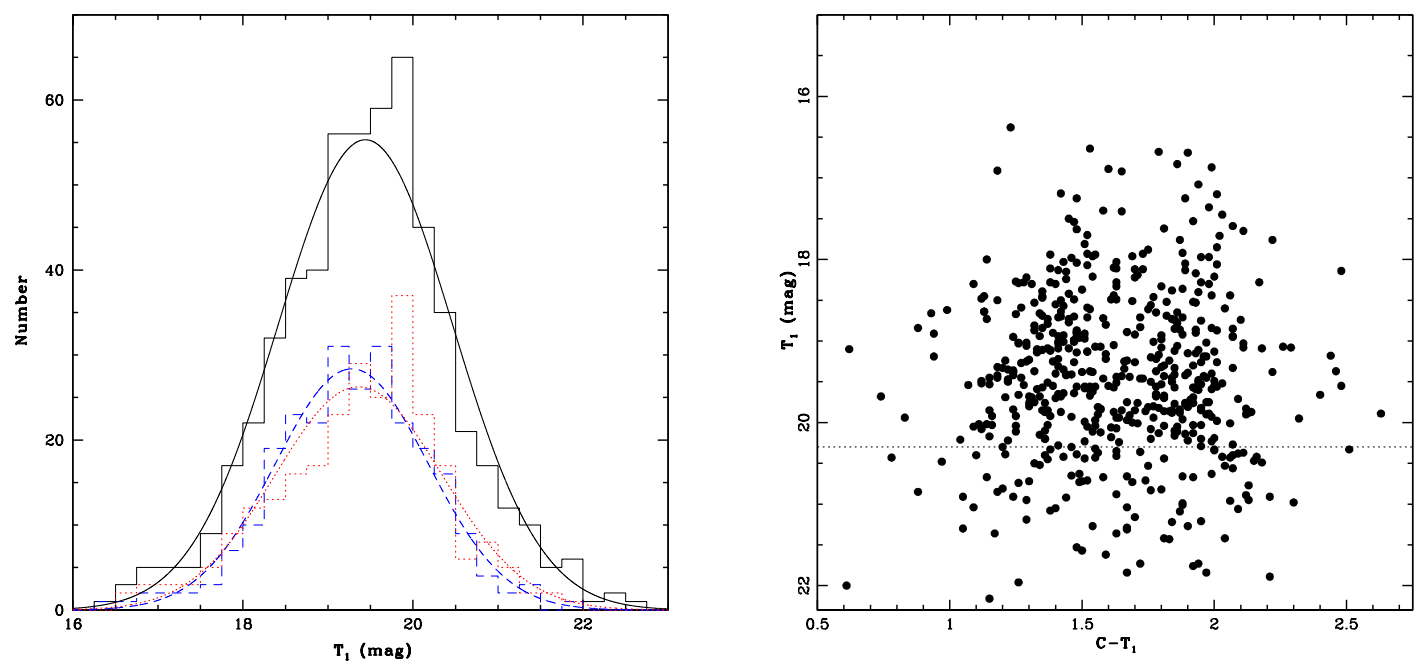

Fig. 7.- Left: The luminosity function of all GCs in NGC 5128 with available $\mathrm{T}_{1}$ magnitude $(\mathrm{N}=569)$ is fit by a Gaussian function with a mean of $19.44 \pm 0.04 \mathrm{mag}$ and sigma $=1.03 \pm 0.03 \mathrm{mag}$. The luminosity functions for the metal-poor (blue) and metal-rich (red) GCs are also fit by Gaussian functions with means of $19.28 \pm 0.06 \mathrm{mag}$ and $19.36 \pm 0.06 \mathrm{mag}$ and sigmas of $0.90 \pm 0.04 \mathrm{mag}$ and $0.98 \pm 0.04 \mathrm{mag}$ respectively. Right: The color-magnitude diagram of the entire GCS in NGC 5128 exhibits the color-bimodality of blue and red GCs. The dotted line represents the expected turnover magnitude. See the electronic version for the color figure. 


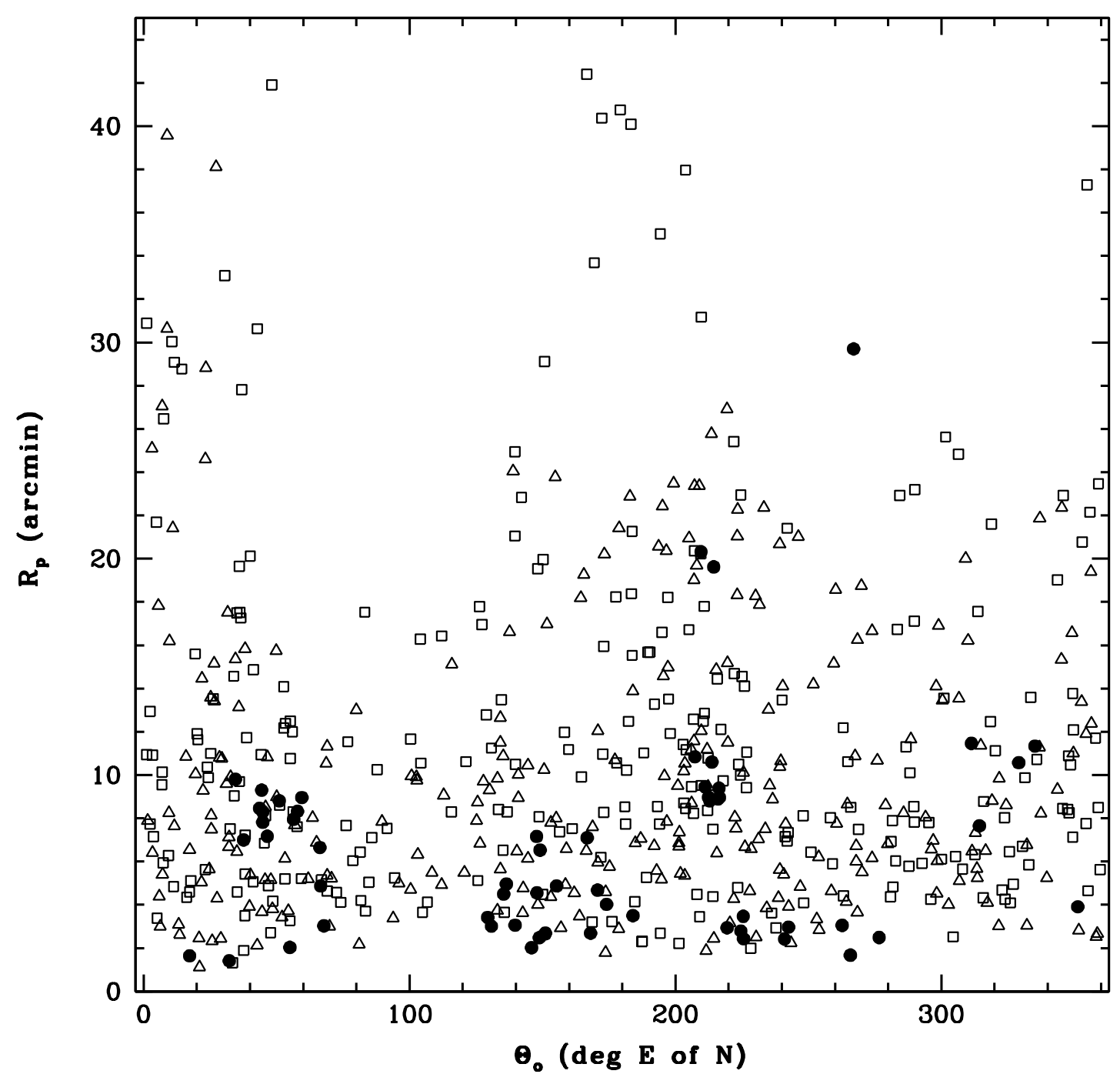

FIG. 8. - The GCS of NGC 5128 is shown as their galactocentric radial position as a function of their azimuthal position measured in degrees East of North on the projected sky. The squares are the metal-poor GCs with a measured velocity, the triangles are metal-rich GCs with a measured velocity, and the solid circles are GCs that either have measured velocities but no color information, or GCs with no measured radial velocities. These latter objects were confirmed from resolved Hubble Space Telescope images (Harris et al. 2006). Clearly, the outer regions $\left(>10^{\prime}\right)$ of NGC 5128 need to be searched for GCs to remove any potential spatial bias in this region. 


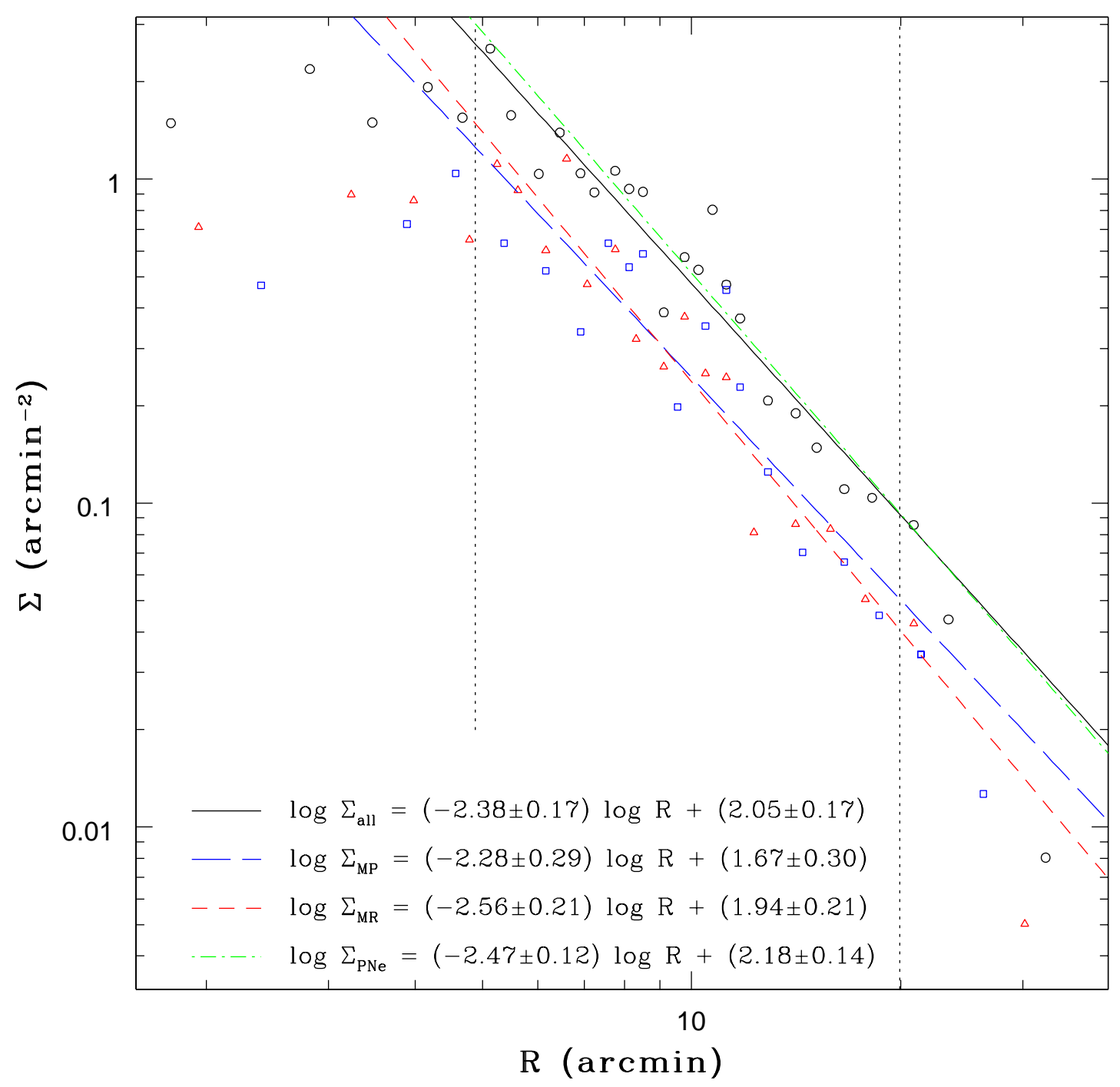

FIG. 9.- The surface density profiles of the GCS is shown binned in circular annuli of equal numbers for the entire GCS (black circles), the MP GCs (blue squares), and the MR GCs (red triangles). These three populations were fit with a power law function between the regions of azimuthal coverage between $5^{\prime}-20^{\prime}$ indicated by the horizontal dashed lines. The best fits are labelled, and shown for the entire GCS (black line), MP GCs (long dashed blue line), and MR GCs (short dashed red line), respectively. The PNe power law fit between $5^{\prime}-20^{\prime}$ is also shown (green dashed dotted line). See the electronic version for the color figure. 


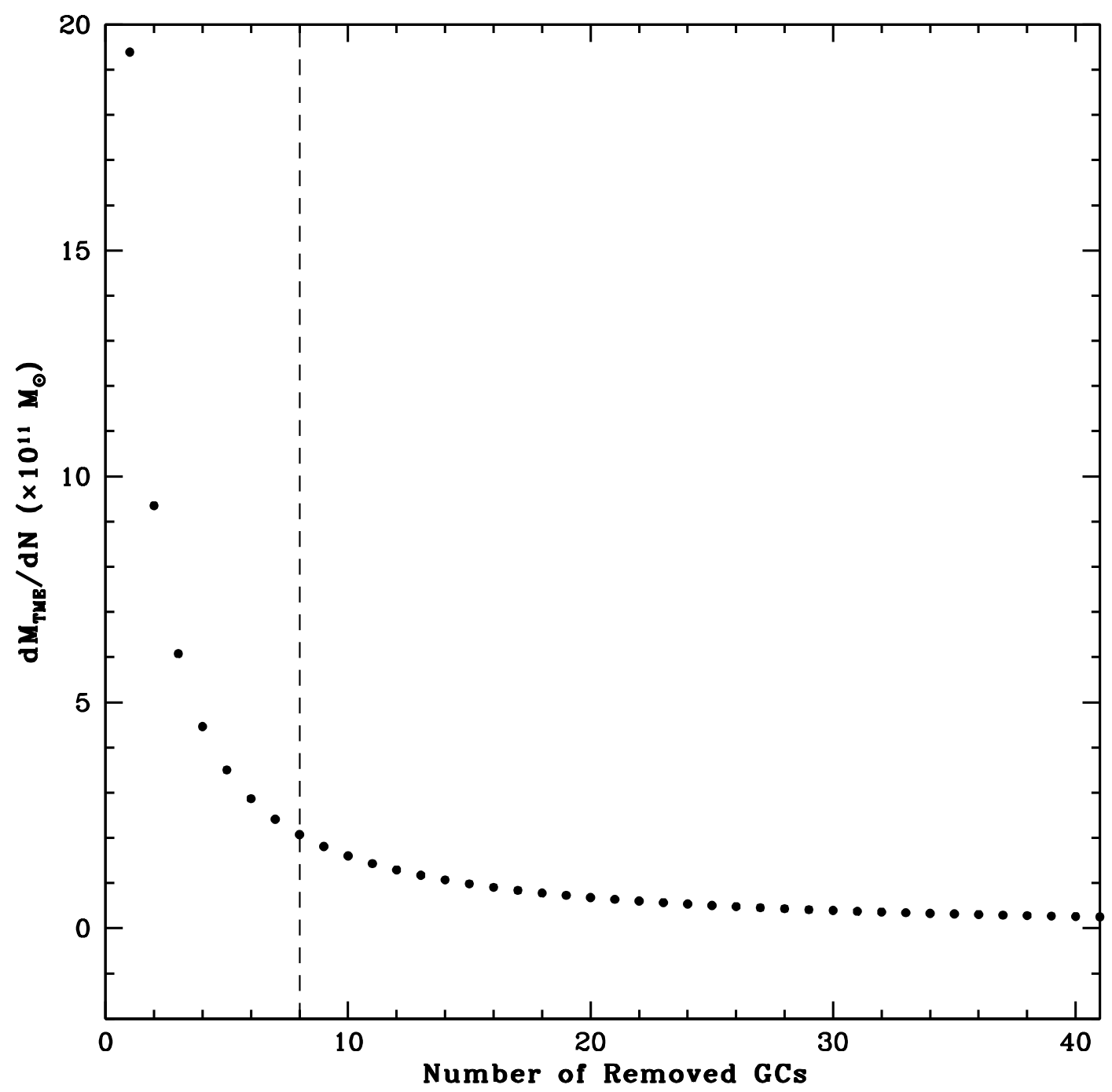

FIG. 10.- The total change in mass normalized by the number of GCs subsequently removed with decreasing $v^{2} R$ contribution in the Tracer Mass Estimator, is shown. We selectively remove $8 \mathrm{GCs}$ with the largest $v^{2} R$ contribution indicated by the dashed line, which inflate the total mass estimate of NGC 5128. 


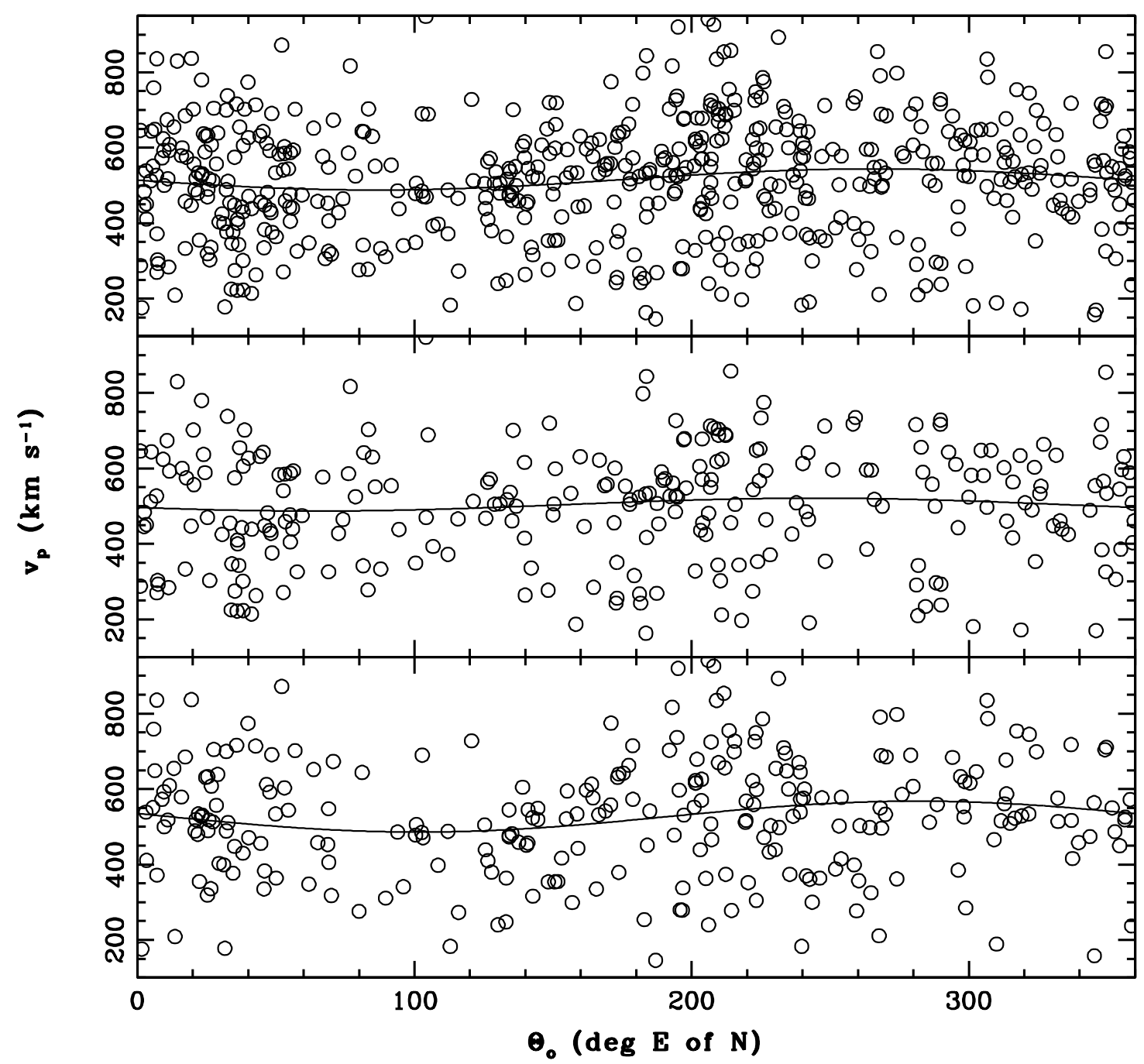

FIG. 11. - The rotation axis and measured radial velocity are shown for the entire GC sample (top panel), the metal-poor sample (middle panel), and the metal-rich sample (bottom panel). The GCs have been fit with Equation 8 which is overplotted as the solid curve. The fitted parameters are listed in Table 6 . 

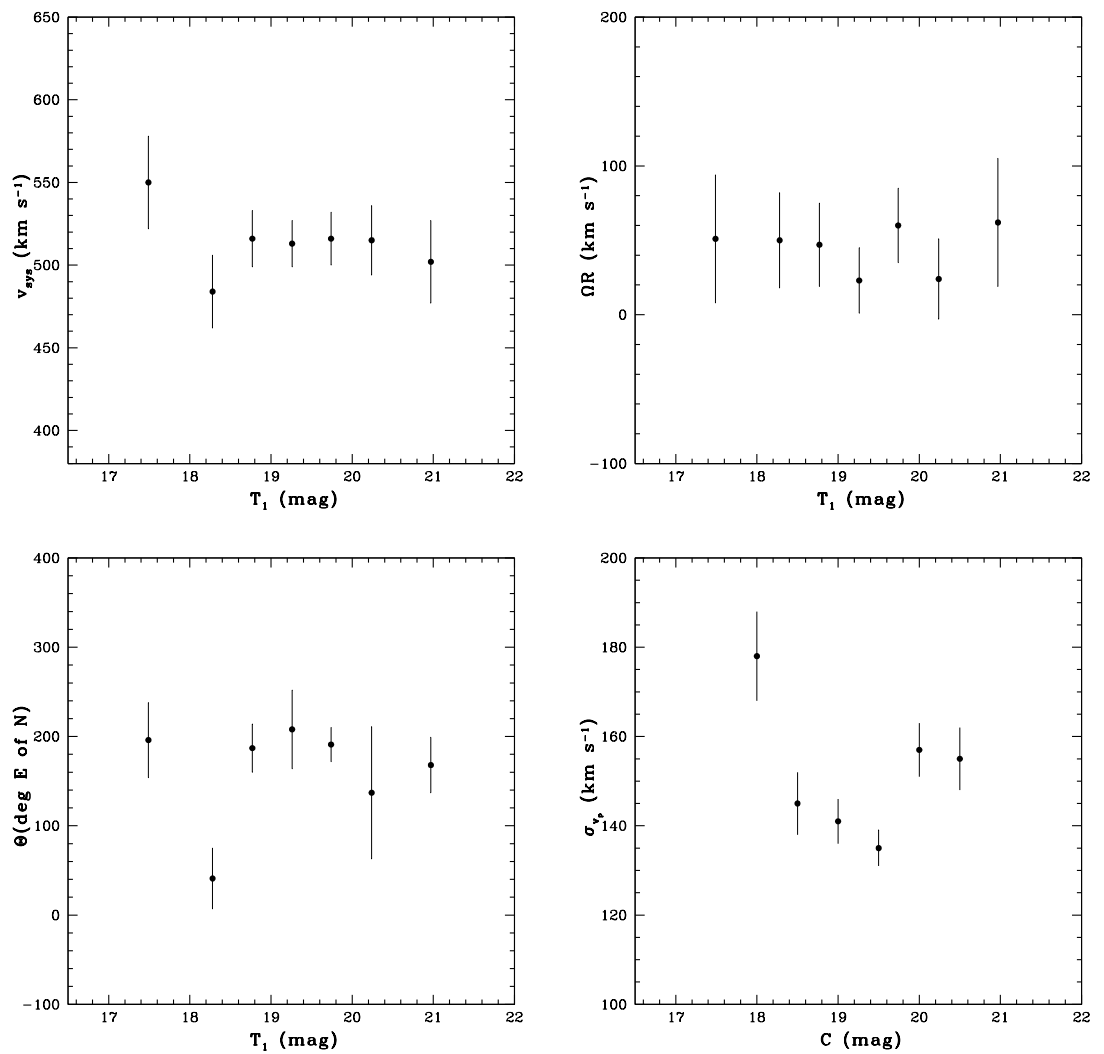

FIG. 12.- The $v_{\text {sys }}$ (top left panel), $\Omega R$ (top right panel), $\Theta_{0}$ (bottom left panel), and $\sigma_{v_{p}}$ (bottom right panel) are plotted as a function of $\mathrm{T}_{1}$ magnitude. 